\title{
Korpuslinguistische Perspektiven auf die sprachhistorische Entwicklung der nominalisierten Infinitive im Deutschen
}

\author{
Martina Werner (Wien)
}

\begin{abstract}
Nominalized infinitives (NIs, such as (das) Lachen '(the) laughing', (das) Um-die-Ecke-Wohnen lit. '(the) around-the-corner-living') are the morphologically most elaborated deverbal nominalization patterns without restrictions in present-day German. However, this was not the case in earlier stages of German. The article reconstructs the steps behind the increase in productivity of the NI since Old High German with the support of historical corpora from Old High, Middle High, Early New High, and present-day German. It will be shown that the increase in productivity is due to an interplay of morphology and syntax. Syntactically, NIs develop from the verbal infinitive together with a syncretism with the pre-Old High German nominal gerund. Morphologically, NIs replace -ung-derived nouns in order to form abstract nouns in Middle High German. In a further step, the morphological complexity of NIs increases, because syntagmata are nominalized, including the emergence of synthetic compounds. In a reverse development taking place from Middle High German onwards, nominalized infinitives can also get result-readings which coincides with pluralizability (such as das Schreiben 'letter' - die Schreiben 'letters').
\end{abstract}

\section{$1 \quad$ Ausgangspunkt: Was sind Infinitive?}

Infinitive ${ }^{1}$ gelten sowohl typologisch als auch wissenschaftshistorisch in der Linguistik seit jeher als morphosyntaktisch hybride Erscheinung, die innerhalb der Wortartklassifikation zwischen Nomen und Verb angesiedelt ist (cf. bereits Jolly 1873, jüngst Hentschel 2017). ${ }^{2}$ Verbal sind Infinitive als Teil eines Verbalkomplexes, ${ }^{3}$ bei der Verwendung mit der Infinitivpartikel zu (zu lesen, zu lachen, optional mit modifizierenden Adverbien, cf. schnell zu fahren, langsam zu essen) sowie bei Beibehaltung der Komplemente (wie in Pilze im Wald zu suchen ist

\footnotetext{
${ }^{1}$ Dieser Beitrag ist im Rahmen des Elise-Richter-Forschungsprojekts „Diachronie des substantivierten Infinitivs“ (V-347 G23) sowie des Einzelprojekts „Relationale Adjektive in der Sprachgeschichte des Deutschen (P32415), beide gefördert vom FWF, entstanden.

${ }^{2}$ Zur Auffassung, wonach der Infinitiv in den idg. Sprachen ein Verbalsubstantiv ist, cf. zuerst Bopp (1833). Die Auffassung Sandbergs (1976), wonach Infinitive etymologisch aufgrund der nominalen -en-Markierung diachron wie synchron der Derivation zuzuordnen sind, ist hingegen unplausibel, da die -en-Markierung neben dem nominalen auch beim verbalen Infinitiv auftritt (zu einer detaillierten Diskussion cf. Schlotthauer/Zifonun/Cosma 2014: 271-273), so auch bereits im Althochdeutschen (cf. Abschnitt 2), und somit keine exklusiv nominale Markierung darstellt.

${ }^{3}$ Zur Syntax des Infinitivs cf. erstmals Bech (1955/1957) sowie aktueller Blume (2004).
} 
gefährlich), die für den nominal(isiert)en Infinitiv nicht möglich ist, da hier nur Attribute (wie in das Suchen der Pilze/von Pilzen) angeschlossen werden können (Gaeta 1999: 178-180). Nominal sind die Infinitive des Gegenwartsdeutschen, wenn sie mit Determinatoren (dieses Lachen, das Herumirren) oder mit Attributen (kurzes Spazierengehen, Bellen des Hundes) auftreten. Da die sog. bare infinitives, also die unmarkierten Infinitive (wie in Irren ist menschlich), sich auf zweierlei Arten auflösen lassen (verbal: $Z \boldsymbol{u}$ irren ist menschlich; nominal: Das Irren ist menschlich), argumentiert Bierwisch (2009, ähnlich hierzu jüngst Iordăchioaia 2019) dafür, zwei Arten von Infinitiven, einen verbalen und einen nominalen Typ, anzusetzen. Leiss (2002) folgert stattdessen daraus, dass Infinitive eine Kategorie darstellen, die sowohl nominale als auch verbale Eigenschaften besitzt. Infinitive sind ihr zufolge als wortartneutral einzustufen und die jeweilige Interpretation als verbal bzw. nominal erfolgt auf syntaktischer Ebene über Monosemierung. Verglichen wird dies mit der Diskussion um die Wortartzugehörigkeit von Partizipien, die als Verben bzw. Adjektive klassifiziert werden (cf. hierzu auch Fuhrhop/Teuber 2000). Auch die relationalen Adjektive (wie in richterliche Entscheidung) wären als eine solche ianusgesichtige Wortart zu klassifizieren. Hierzu werden folgende Argumente geltend gemacht: fehlende Komparierbarkeit (cf. * richterlichere Entscheidung) und Nominalisierbarkeit (wie in * Richterlichkeit) sowie Mangel an Verwendbarkeit in Prädikativkonstruktionen (wie in * Sie ist richterlich) und Negation (wie in *unrichterliche Entscheidung (zu Details cf. z. B. Gunkel/Zifonun 2008, Zifonun 2011). Bei entsprechendem Gebrauch kippen die relationalen Adjektive in den genannten Konstruktionen in eben genau diese qualitative Semantik sekundär (umgekehrt hierzu Frevel/Knobloch 2005). Da sie semantisch typischerweise Thetarollen zu einem deverbalen Bezugsnomen kodieren (wie bei richterliche Entscheidung ist Richter Subjekt; cf. sprachvergleichend Marchis Moreno 2015) entsprechen die relationalen Adjektive nicht dem Prototyp eines (Eigenschafts-)Adjektivs, sondern sind ebenfalls an der Schnittstelle von Nominalität und Adjektivität anzusiedeln.

Dass der NI an der Schnittstelle von Nominalität und Verbalität angesiedelt ist, zeigt auch der Sprachvergleich: In der Sprachgeschichte des Englischen bspw. ist die Infinitivendung zum Frühneuenglischen hin geschwunden (Tanaka 2007: 53-55), als Folge davon sind auch im Gegenwartsenglischen Infinitive endungslos (to cut, to slap) bzw. formal nicht unterscheidbar von nominalen Stammkonversionen ( $a$ cut, a slap), die mereologisch aufgrund ihrer Pluralisierbarkeit mit der markierungslosen Perfektivität englischer Verben, welche zur Imperfektivitätserzeugung bekanntlich die -ing-Progressivform bilden, parallelisiert werden können (cf. auch Vogel 1996). Umgekehrt ist im Englischen aus einem ehemaligen Nominalisierungsmuster des altenglischen -ing-Suffixes über Synkretismus mit dem -end(e)-Partizip Präsens die heutige ing-Form entstanden (zu Details der Rekonstruktion cf. Moessner 1997, Alexiadou 2013), die nominal (wie bei the dancing) und verbal (wie bei John is dancing) verwendet werden kann. In einer Sprache mit geringer, d. h. v. a. syntaktisch gesteuerter, Nomen/Verb-Distinktion wie dem Englischen spricht man daher von ,Konversion“ beim unmarkierten Wortartwechsel, während für Sprachen mit morphologisch markierter Nomen/Verb-Distinktion wie dem flektierenden Deutschen der Terminus ,grammatische/syntaktische Transposition' vorgeschlagen wird (cf. hierzu Vogel 1996 zum Forschungsüberblick). Dem Vorschlag Vogels soll in diesem Beitrag gefolgt werden. 
Während die Etablierung des Musters eines nominalisierten Infinitivs in anderen germanischen Sprachen wie dem Gotischen oder Altnordischen zu keiner Entfaltung kam (Jolly 1873: 173), ist dies im Deutschen anders: Bereits aus flexionsmorphologischer Sicht kann der Infinitiv im Deutschen als Substantiv eingeordnet werden (cf. Schlotthauer/Zifonun/Cosma 2014: 257f.), cf. das Flexionsverhalten bei Kasus (wegen des Rauchens). ${ }^{4}$ Da im vorliegenden Artikel gerade der Frage der Transposition und sukzessiven Integration des Infinitivs in den nominalen Bereich nachgegangen wird, soll der Annahme von Leiss (2002), wonach der Infinitiv eine wortartunterspezifizierte Kategorie ist, gefolgt werden. Hinsichtlich der „Vererbungsprozesse“ aller verbspezifischen (wie Aspekt/Tempus, Genus Verbi, Modus/Modalität ${ }^{5}$ ) bzw. nomenspezifischen grammatischen Kategorien (wie Genus, Numerus, Kasus) in die jeweils andere Domäne schlussfolgern Alexiadou/Iordăchioaia/Schäfer (2011) im Vergleich von Spanisch, Rumänisch, Deutsch und Englisch, dass die kategoriellen Vererbungsmöglichkeiten einzelsprachlich geregelt sind. Dies muss im Rahmen des vorliegenden Beitrags einer gesonderten empirischen Untersuchung zu Vererbungsprozessen verbaler Kategorien bei der Nominalisierung des Infinitivs vorbehalten bleiben, wird jedoch aus sprachhistorischer Sicht in Abschnitt 5.2 bei der Diskussion der Nominalisierung reflexiver Verben aus flexionsmorphologischer Sicht forschungsüberblickshaft mitberücksichtigt.

\subsection{Aufbau und Methodologie des Beitrags}

Vor dem skizzierten Hintergrund ist der vorliegende Artikel daher folgendermaßen aufgebaut: Zunächst wollen wir uns die Distribution von nominalisierten Infinitiven im Gegenwartsdeutschen ansehen (1.2), bevor die weiteren Kapitel 2-5 in die Sprachgeschichte blicken. Aus diachroner Sicht ist dabei zunächst die Frage von Bedeutung, welche Schritte zur Nominalisierung des Infinitivs in der heutigen Form beigetragen haben. Dazu beleuchtet Kapitel 2 den Ursprung des NIs und Kapitel 3 dessen Entwicklung aus der Syntax. Kapitel 4-5 nehmen den NI aus morphologischer Perspektive in den Blick: Dabei soll zuerst die Motivation der Produktivwerdung des NIs innerhalb der Dynamik deverbaler Nominalisierungsmuster (Kapitel 4) im Vordergrund stehen. Anschließend wird in Kapitel 5 der Blick auf die Nominalisierung von Infinitivphrasen (Rolle der Argumentstruktur in 5.1, reflexive Verben in 5.2) gelegt werden, bevor Bezügen zur Lexikalisierung in Abschnitt 6 nachgegangen wird. Behaghel (1923: 356) zufolge gilt ein Infinitiv als nominalisiert, wenn er konkrete Bedeutung besitzt wie in dem Satz Das

\footnotetext{
${ }^{4}$ Für die Distribution von Absentiv, Progressiv und der beim-x-sein-Konstruktion ist Gegenstand der Diskussion, ob die involvierten Infinitive nominal oder verbal sind (cf. Engelberg 2004, 2008). Die Infinitive der v. a. in den Dialekten und heutigen Regiolekten gebräuchlichen tun-Periphrase (Typ: Er tut gerade singen) geht historisch zurück auf einen nominal gebrauchten Infinitiv (Behaghel 1923: 361). Synchron lässt sich der Infinitiv des Absentivs (Hans ist essen) als nominal identifizieren, während dies für den Progressiv (am essen sein) und für die beim-Konstruktion (wie beim essen sein) nicht zutrifft (Engelberg 2008: 14). Da es hier aber um den Gebrauch der NIs außerhalb von Verbalperiphrasen geht, soll die Verwendung von NIs innerhalb von verbalen Periphrasen im vorliegenden Beitrag nicht betrachtet werden. Für eine gesonderte Betrachtung von NIs in verbalen Periphrasen sollten dabei auch die Funktionsverbgefüge mitberücksichtigt werden, da bei ihnen der NI ebenfalls vorkommt (wie ins Stocken geraten, ins Rollen kommen).

5 Terminologisch wird insbesondere in der älteren Literatur nicht unterschieden zwischen Modus und Modalität. Der NI kann im Gegenwartsdeutschen jedoch nie im Konjunktiv vorkommen (das *Essen-Würden, das *BesuchtHätten), ist im Deutschen also assoziiert mit der verbalen Finitheit.
} 
Essen steht auf dem Tisch. Damit einhergehende Bedeutungsveränderungen und ihre Frequenz im Gegenwartsdeutschen sollen in diesem Abschnitt also eingehender betrachtet werden.

Um dem Forschungsdesiderat einer stärkeren Vernetzung empirischer und theoretischer Befunde Rechnung zu tragen, wurden Datenerhebungen zu verschiedenen Sprachstufen des Deutschen mit den theoretischen Befunden aus der Literatur kombiniert. Dazu wurden Belege aus den folgenden Korpora und unter Konsultation folgender historischer Wörterbücher verwendet: für Althochdeutsch (Ahd.) das Korpus Deutsch Diachron Digital (DDD), für Mittelhochdeutsch (Mhd.) das Referenzkorpus Mittelhochdeutsch (REM). Da der Umfang der Distribution bzw. die Produktivität des NIs besonders für das Ahd. und Mhd. bislang unbekannt war, wurden das DDD und das REM einer genauen empirischen Überprüfung unterzogen. Bei ambigen Fällen wurde zudem das mhd. Wörterbuch von Lexer und Benecke/Müller/Zarncke (BMZ), die Mittelhochdeutsche Begriffsdatenbank (MHDBDB), das frühneuhochdeutsche (fnhd.) Wörterbuch FWB sowie das Grimm'sche DWB verwendet.

$\mathrm{Ab}$ fnhd. Zeit gilt die Produktivität des NIs nicht nur als gesichert, sondern nimmt geradezu sprunghaft zu, auch was die innere morphologische Struktur der Wörter anbelangt (cf. Kapitel 4, 5). Um die hohe Datendichte und -vielfalt empirisch bewältigbar zu machen, konzentriert sich die vorliegende Untersuchung im Fnhd. und Neuhochdeutschen (Nhd.) auf eine qualitative Auswertung, um aus den Befunden deskriptive Generalisierungen abzuleiten. Hierfür wurde für das Fnhd. und Nhd. das Deutsche Textarchiv (dwds.de) verwendet. Für die Gegenwartssprache (Ende 20./Anfang 21. Jh.) wurde neben qualitativen Belegen aus dem DWDS-Kernkorpus reine randomisierte Stichprobe des Austrian Media Corpus genommen. ${ }^{6}$ Weitere Belege stammen aus dem Datenmaterial von Lawrenz (2006) zur Phrasenkomposition.

\subsection{Ausgangspunkt: Frequenzzunahme}

Um zunächst einmal zu prüfen, ob es diachron tatsächlich zu einer Zunahme von NIs kam, wurden das Korpus DDD für das Ahd. und das Korpus REM für das Mhd. einer empirischen Auswertung unterzogen. Für das DDD und REM wurde dabei jeder Infinitiv als NI gewertet, wenn dieser mit einem Determinator oder syntaktisch als Nomen verwendet wurde (z. B. als Attribut wie in Anzeige wegen Fahrens ohne Helm). Da subjektwertige Infinitive (wie in Irren ist menschlich) sowohl verbal (Zu irren ist menschlich) als auch nominal (Das Irren ist menschlich) auflösbar sind (cf. Abschnitt 1), wurden diese, soweit keine anderen Evidenzen wie etwa Attribute desambiguierend fungieren konnten, in der Untersuchung nicht berücksichtigt.

Außerdem wurden jeweils nur die Texte in die Untersuchung miteinbezogen, die sich eindeutig einem Jahrhundert zuordnen ließen. Dies wurde für jeden Korpustext einzeln mittels Handschriftencensus überprüft. Auch wenn sich damit für das 10. sowie die zweite Hälfte des 14. Jh. empirisch eine Lücke ergibt, da im DDD für den genannten Zeitraum nur Texte des 10./11. Jh. zur Verfügung stehen, lässt sich unter Zuhilfenahme der restlichen Jahrhunderte trotzdem

\footnotetext{
${ }^{6}$ Für den fachlichen Austausch und Anregungen möchte ich mich bei Patrick Brandt, Gianina Iordăchioaia, Helmut Weiß, Sonja Zeman sowie den anonymen Gutachtern bedanken sowie bei Nina C. Rastinger für formale Hilfe. Bei der korpuslinguistischen Untersuchung haben Arthur Schodterer, Sophia Beiter sowie Hannes Pirker (ACDH$\mathrm{CH})$ mitgewirkt. Für alle möglichen Fehler ist selbstverständlich die Verfasserin verantwortlich.
} 
insgesamt eine deutliche Zunahme der NIs erkennen. Dies gilt insbesondere, da in Abbildung 1 nur die 1 . Hälfte des 14 . Jahrhunderts mitberücksichtigt ist.

Für den Zeitraum 800 bis 1350 lässt sich eine Frequenzsteigerung der NIs feststellen. Zur Ermittlung der jeweiligen Beleganzahlen wurde pro Jahrhundert die Anzahl der Nomina ermittelt. Die relativen Zahlen pro Jahrhundert beziehen sich also auf den jeweiligen Prozentanteil der Zielkonstruktion NI pro Jahrhundert. Für das 13. Jh. sind z. B. 0,47\% aller Nomina substantivierte Infinitive. Die diachrone Zunahme des NIs ist in Abbildung 1 dargestellt. Der Mittelwert aller Belege im diachronen Verlauf wird mit der gepunkteten Linie dargestellt. Vor dem Hintergrund der vorliegenden Daten aus dem DDD und REM, beginnend im 9. bis zur ersten Hälfte des 14. Jh., zeigt die Grafik eine kontinuierliche Zunahme von NIs innerhalb aller Substantive $(n=405.463)$ für diesen Zeitraum. ${ }^{7}$ Wäre zudem die zweite Hälfte des 14 . Jh. graphisch abbildbar, so ist für das 14. Jh. insgesamt ein noch stärkerer Anstieg zu erwarten.

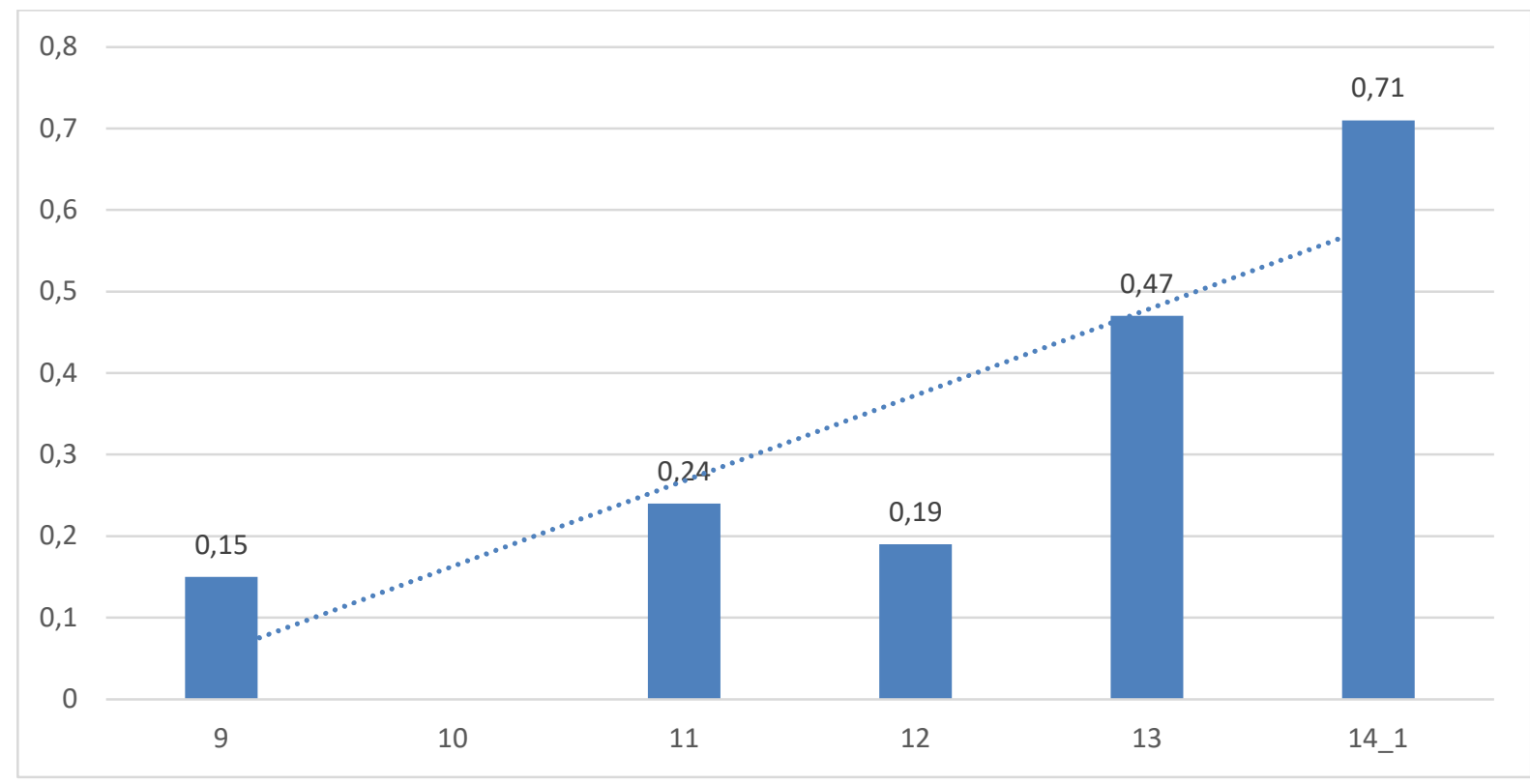

Abbildung 1: Diachrone Zunahme der Zielkonstruktion NI (in \% für alle N pro Jh.) für das Ahd. (DDD) und Mhd. (REM)

Tatsächlich zeigt sich bereits für das 13. Jh. im Vergleich zum 12. Jh. ein deutlicher Anstieg von NIs, der im 14. Jh. (14_1 in der Graphik) fortgesetzt wird. Die Befunde stützen empirisch die Aussage Kloockes (1974), wonach die NIs im 13./14. Jh. eine Blüte in ihrer Produktivität, verglichen mit den vorherigen Jahrhunderten, erfahren.

Den NIs des Gegenwartsdeutschen kommt im Gegensatz zu den zunehmend formal restringierten -ung-Nominalisierungen (cf. *Laufung, *Lachung, *Verschwindung) die Funktion der deverbalen Abstraktnominalisierung zu (Demske 2000, Werner 2012, Hartmann 2014). Um dies für das Gegenwartsdeutsche empirisch zu konkretisieren, wurde eine randomisierte Stichprobe

\footnotetext{
${ }^{7}$ Die Substantivzahlen verteilen sich auf die Jahrhunderte wie folgt: 9. Jh. 34126 Ns (52 Tokens NIs), 11. Jh. 28180 Ns (67 Tokens NIs), 12. Jh. 85828 Ns (165 Tokens NIs), 13. Jh. 142973 Ns (670 Tokens NIs) sowie für die 1. Hälfte des 14. Jh. 114356 Ns (815 Tokens NIs). Die Korpusabfrage ergab keine für das 10. Jh. chronifizierbaren Daten.
} 
aus dem Austrian Media Corpus (AMC) ${ }^{8}$ genommen, welches Texte von 1987-2017 enthält (insg. 10.549.292.505 Tokens, 7.769.944.139 Wörter). Die gewählte Stichprobe umfasste 58.084 Wörter ( $\mathrm{n}=58.084$ running words). Der NI lässt sich unter allen Substantiven zu 1,62\% belegen. Zum Vergleich: Suffigierungen auf -keit lassen sich „lediglich“ zu 0,52\% und auf heit zu 0,50\% belegen. Im Gegensatz dazu sind -ung-Nominalisierungen zwar deutlich häufiger (4,43\% aller Nomina), allerdings sind hierunter sehr oft auch die gerade erwähnten Lexikalisierungen (wie Heizung, Wohnung) bzw. Substantive mit ambiger Referenz (wie z. B. Bedienung ,Aktivität, etwas/jmd. zu bedienen“ sowie ,Person, die bedient') enthalten. In einer früheren Untersuchung (cf. Duden 1995: 299) ist der NI das zweithäufigste Nominalisierungsmuster nach den -ung-Nominalisierungen. Letztere unterliegen allerdings formalen und semantischen Restriktionen, so dass dieses Ergebnis synchron wie diachron deutlich zu relativieren ist: So sind -ung-Nomina nicht erst im Gegenwartsdeutschen, sondern bereits seit fnhd. Zeit (Demske 2000) zunehmend beschränkt auf die Nominalisierung perfektiver Verben, cf. *Tretung, ${ }^{*}$ Lassung, *Drückung aber Betretung, Auslassung, Bedrückung. Auch aus semantischer Sicht sind im Gegenwartsdeutschen viele der -ung-Bildungen lexikalisiert, d. h. dauerhaft bedeutungsverengt auf eine der Dimensionen Agens, Resultat, Lokativ oder Instrument (Ehrich/Rapp 2000, Lübbe/Trott 2017: 291). Somit kann für das Gegenwartsdeutsche formuliert werden, dass eines der häufigsten Nomina kein typisches nominales Simplex, wie man vielleicht erwarten würde, sondern der bzgl. einer konkreten Wortarteinordnung ambige substantivierte Infinitiv ist.

Im diachronen Kontrast zwischen den Anfängen des NIs im Alt- und Mittelhochdeutschen in blau (DDD zum Ahd., REM zum Mhd.) vs. dem Gegenwartsdeutschen (randomisierte Stichprobe aus dem AMC) in grün ist die Produktivitätszunahme deutlich zu erkennen, cf. Abbildung 2.

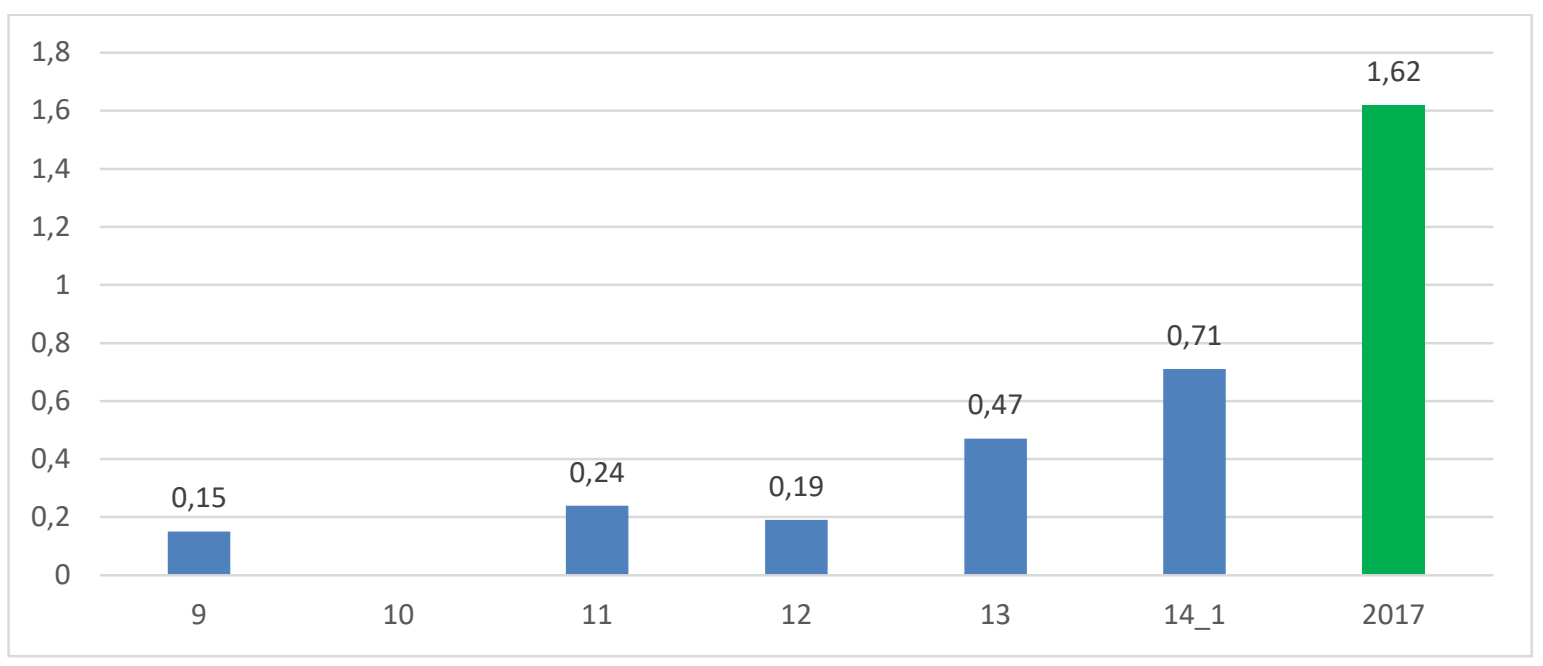

Abbildung 2: Diachrone Zunahme der Zielkonstruktion NI (in \% für alle N pro Jh.) für das Ahd. (DDD) und Mhd. (REM) im Vergleich zum Gegenwartsdeutschen (AMC in grün) ${ }^{9}$

\footnotetext{
${ }^{8}$ Das Austrian Media Corpus am Austrian Centre for Digital Humanities and Cultural Heritage enthält alle Presseerzeugnisse der Österreichischen Presse Agentur APA (1987-2017). Um zu gewährleisten, dass aus der großen Datenmenge eine möglichst repräsentative für das Gegenwartsdeutsche selegiert wird, wurde eine randomisierte Stichprobe aus dem Jahr 2017 anhand eines arbiträren Kriteriums erzeugt. Hierfür wurden in diesem Fall alle Texte gewählt, die mit dem arbiträr gewählten Wort der bzw. Der beginnen.

${ }^{9}$ Die Korpusabfrage ergab keine für das 10. Jh. chronologisierbaren Daten.
} 
Unter den deverbalen Abstraktnominalisierungen kann der NI im Gegenwartsdeutschen sowohl als das morphologisch als auch lexikalisch (d. h. im Hinblick auf verschiedene Verbklassen) restriktionsloseste (zu Details cf. Blume 2004, Lübbe/Trott 2017) und damit produktivste Nominalisierungsmuster für deverbale Abstrakta gelten. Da dies nicht immer so war, begibt sich der folgende Abschnitt zunächst zur „Ursuppe“ des NIs, also zu den Anfängen seiner Produktivwerdung im Althochdeutschen.

\section{Zum Ursprung der nominalisierten Infinitive}

Der Ursprung des NIs galt lange Zeit als umstritten. Kloocke (1974) nahm für das Mhd. einen Einfluss des Mittelfranzösischen an (wie nfrz. le sourire, das Lächeln', le souvenir ,Andenken, Erinnerung'). Allerdings ist der NI im Französischen ${ }^{10}$ - im Gegensatz zum Italienischen, Portugiesischen und Spanischen - in der Standardsprache praktisch unproduktiv, d. h. es treten synchron Restriktionen auf (z. B. *le lire ,das Lesen', *le penser, das Denken'). Der Produktivitätsabbau des französischen NIs begann im 13. Jh. im Zusammenhang mit der Entwicklung von VO (zu Details der Rekonstruktion cf. Sleeman 2010, Meinschäfer 2016: 10f. und die dort zitierte Literatur). Dass die Sprachkontakthypothese nicht nur aus chronologischen Gründen nicht zwingend ist, soll im Folgenden gezeigt werden. Man kann nämlich für die Entstehung des NIs auch eine andere, sprachinterne Quelle des Deutschen annehmen.

Bereits früh (Grimm 1826/II: 595-59, Erdmann 1886: 8f., Wilmanns 1906: 123) wurde in diesem Zusammenhang darauf hingewiesen, dass das Entstehen nominaler Infinitive mit einem Verlust der Infinitivpartikel $z u$ in Zusammenhang steht. Einer alternativen Auffassung zufolge ist der „reine“ (Jolly 1873: 159-161), also wortartunterspezifizierte Infinitiv (wie predigen), bis ins Ahd. üblich und differenziert sich schließlich in einen verbalen (zu predigen) und einen nominalen Infinitiv (das Predigen). Zwischen den genannten Positionen muss allerdings kein Widerspruch bestehen: So kann der Abbau sowohl des unterspezifizierten als auch des verbalen Infinitivs mit $z u^{11}$ in nominalen Kontexten aus syntaktischer Sicht tatsächlich die Entstehung nominal(isiert)er Infinitive (mit-)initiiert haben. Auch dürfte die zunehmende Artikelobligatorik bei Nomina seit ahd. Zeit die syntaktisch nominal verwendeten, „reinen“ Infinitive (wie in Rauchen verboten) zusätzlich begünstigt, $\mathrm{d}$. h. ihre Nominalität gestärkt, allerdings nicht ausgelöst haben (cf. hierzu synchron nämlich Bierwisch 2009: 317), da Nominalität keinen Artikel braucht (schnelles Lesen, Essen der Melone; zur synchronen Diskussion cf. Schlotthauer/Zifonun/Cosma 2014: 268-270). Ebenso kann die Existenz von ererbten Gerundien wie ahd. wizzan

\footnotetext{
${ }^{10}$ In der philosophischen Fachsprache des Gegenwartsfrz. kann der NI dagegen gelegentlich vorkommen. Ich danke Franz Rainer für diesen Hinweis. Interessanterweise wird für die Entstehung des NIs im Frz. der Einfluss des Deutschen geltend gemacht (zum Forschungsüberblick cf. Sleeman 2010), was allerdings ebenso fragwürdig ist wie umgekehrt, da für die postulierte Zeit (Spätahd./Mhd.) die -ung-Nominalisierung noch das vorherrschende Nominalisierungsmuster war. Aus den genannten Gründen sollte m. E., bei aller karolingisch-linguistischen Faszination, die „Sprachkontakt-Hypothese“ für diesen Bereich aufgegeben werden, insbesondere auch deswegen, da auch für ein anderes Nominalisierungsmuster des Deutschen, nämlich die -erei-Nominalisierung, lange ein Einfluss des Suffixes aus dem Frz. angenommen wurde, was allerdings mittlerweile als widerlegt gelten kann, da bereits für das Ahd. ein Einfluss des Lateinischen geltend gemacht werden kann (cf. bspw. Hahnfeld 2015).

${ }^{11}$ Ein Beleg eines verbalen Infinitivs mit $z u$, hier als Komplement mit obligatorisch flektiertem Dativ-e des Infinitivs, aus dem REM (11. Jh., M242-G1, 11425-11436) wäre bspw.: Der mine hende leret in uuige ze uberuuindenne mine fiende, ,der meine Hände lehrt, meine Feinde zu besiegen`.
} 
,Wissen oder leban ,Leben' als zusätzliche Unterstützung der entstehenden Produktivitätserhöhung des NIs gelten, hinsichtlich der Behaghel (1923: 356) einen Synkretismus zwischen $z i+$ Infinitiv und $z i+$-enne-/-anne-Gerund (im Sinne eines flektierten Infinitivs; Terminus nach Schrodt 2004: 85) annimmt. Cf. hierzu Beispiele zum Gerund in (1a-b):

(1a) Oba ir hiar findet iawiht thés thaz wirdig ist des lésannes

(,ob ihr hier etwas findet, das würdig ist des Lesens-GEN')

(Otfrid, Evangelienbuch III: 8.7, zitiert nach Demske 2001: 61)

(1b) Nu gárawemes unsih álle zi themo fehtanne

(,Nun rüsten wir uns alle für das Fechten-DAT')

(Otfrid, Evangelienbuch II: 3.55, zitiert nach Demske 2001: 61)

(1c) Uuánda íro gében álso getân íst

(,Weil ihr Geben so gemacht/geartet ist [, dass ... $]^{\circ}$ )

(Notker, De Consulatione II: 45-48, zitiert nach DDD)

(1d) thaz wéinon was in léngi himilo gizéngi;

(,Das Weinen dauerte an, den Himmel erreichend;")

(Otfrid, Evangelienbuch I: 20.10, zitiert nach DDD)

Ahd. Gerundien sind sowohl syntaktisch aufgrund des Auftretens als nominale Konstituente und mit Pronomina als auch semantisch aufgrund ihrer prozessualen Semantik nicht von den nominal gebrauchten Infinitiven zu unterscheiden (cf. auch Daten aus dem DDD, 1c-d), so dass ein Synkretismus beider Formen zu dieser Zeit anzunehmen ist.

\section{Die Entwicklung von Nominalität}

\subsection{Abbau verbaler Rektion}

Zur Entwicklung von Nominalität muss aus Sicht der Transposition auch der Blick auf den unterspezifizierten bzw. verbalen Infinitiv gelenkt werden. Infinitive können noch im Mhd. mit Komplementen verbaler Rektion unter Ausschluss von Interlinearübersetzungen ${ }^{12}$ auftreten (Behaghel 1923: 370f., Koning 1933; Mayerthaler et al. 1993: 137, Vogel 1996: 132-134, Schlotthauer/Zifonun/Cosma 2014: 268), cf. (2): ${ }^{13}$

(2a) dà wart vil michel grüezen die lieben geste getân

(,Da wurde ein sehr großes Grüßen der [wörtl. die] lieben Gäste durchgeführt')

(Nibelungenlied 786: 4, zitiert nach Gaeta 1998: 6)

\footnotetext{
12 Ein Beispiel für eine Interlinearübersetzung aus dem REM (12. Jh., M188y-N1 14173-14184) wäre: in behudinde gechofe dine ,um Dein Wort zu behalten'. Dies ist daran zu erkennen, dass diese Wendung auch aus der englischen Sprachgeschichte bekannt ist, cf. Vespasianischer Psalter in haldinge word din, Canterbury Psalter on to gehaeldenne word pine bzw. frühester englischer Prosa-Psalter in keeping py wordes (Rolle of Hampale in kepand pi wordis), allesamt Lehnübersetzungen aus lat. custodiendo sermones tuos (Weber 1899: lxxv).

13 Eine systematische Untersuchung zu Nominalisierungen mit Komplementen verbaler Rektion hat Panagl (2006) zu den idg. Sprachen vorgelegt. Sie ist im Deutschen nicht auf den NI beschränkt, sondern kommt auch bei Suffigierungen vor, cf. die esser die yngewey der menschen (cf. Behaghel 1923: 723f.) wörtl. , die Esser [die Eingeweide]-AKK der Menschen', also ,die Esser der Eingeweide der Menschen'.
} 
(2b) da machet got ein scheiden die lieben von den leiden

(,Da macht Gott ein Trennen der [wörtl. die] Guten von den Bösen')

(Warnung 3343, zitiert nach Dahl/Eroms 2014: 115)

Das Vorkommen mit einem vom Infinitiv regierten Kasus kann als eindeutiger Beweis für die Verbalität, die Belege mit Adjunkt-Genitiv als eindeutiger Beweis für die Nominalität des Infinitivs gewertet werden. Gaeta (1998) folgert daraus, dass der NI im Mhd. noch stärker verbale Eigenschaften hatte und heute eher nominale Eigenschaften ( $<$ synthetische Komposition) hat. Der Aspekt der synthetischen Komposition soll in Kapitel 5 erneut aufgegriffen werden.

\subsection{Etablierung von Definitheit}

In Bezug auf die Kombination des NIs mit Determinatoren ist es aufgrund der früher einsetzenden Grammatikalisierung des definiten Artikels rein quantitativ wahrscheinlicher, dass die NIs mit diesem zuerst auftreten. Dies lässt sich tatsächlich zeigen (cf. Abbildung 3, blaue Linie): Noch im 11. Jahrhundert sind keine NIs mit indefinitem Artikel (0 Tokens) im REM dokumentiert, sehr wohl aber mit definitem Artikel (9 Tokens).

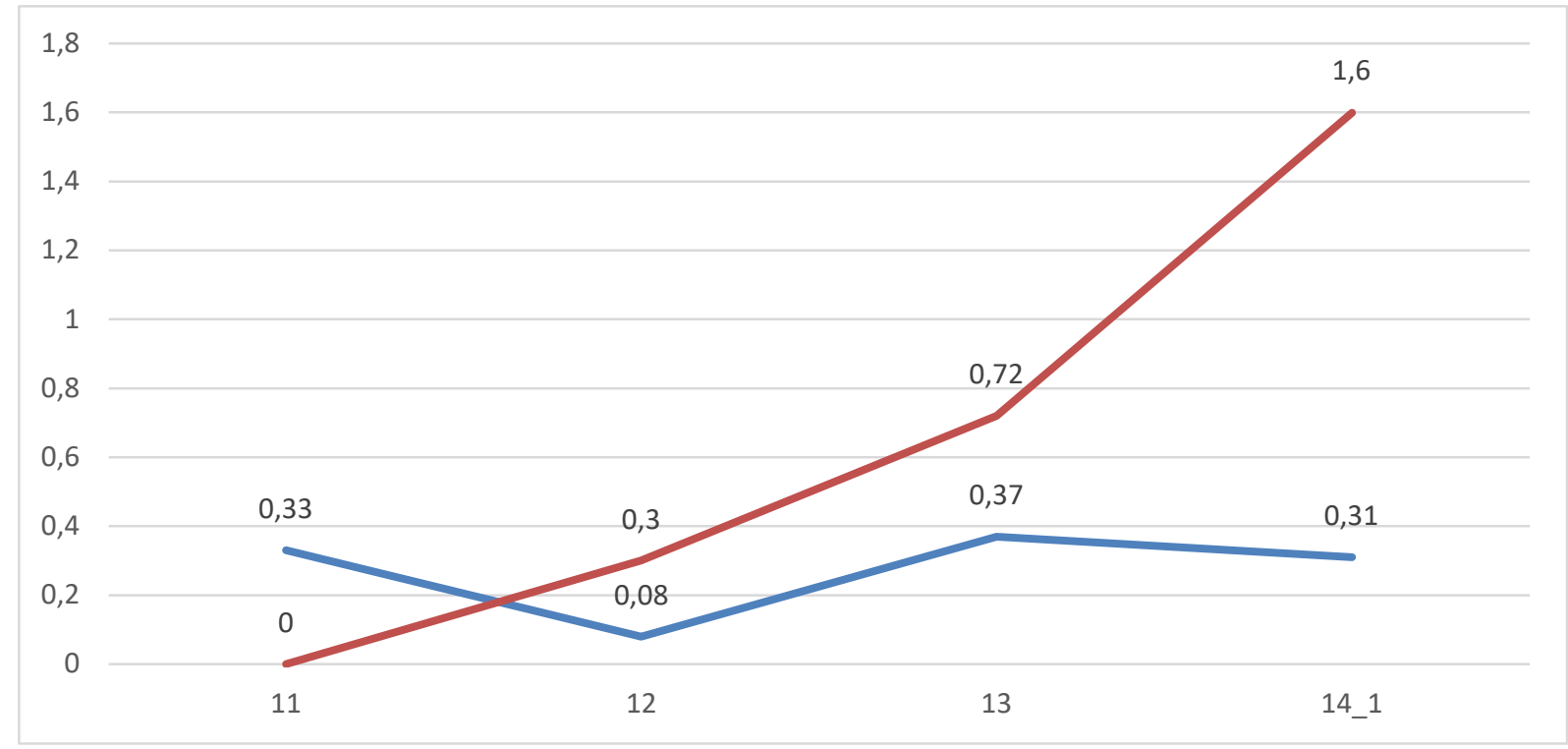

Abbildung 3: Frequenz (Tokens) (in \% für alle N pro Jh.) des definiten Artikels mit NIs (blau) und des indefiniten Artikels mit NIs (orange) im Ahd. (DDD) und Mhd. (REM)

Abbildung 3 zeigt alle definiten bzw. indefiniten Artikel mit NIs (Typ: das/ein Lachen) in Relation zu der Artikelverwendung bei allen Substantiven pro Jahrhundert. Der definite Artikel (blaue Linie) tritt zuerst mit NIs auf. ${ }^{14}$ Inwieweit die Nominalisierung der NIs durch den zunehmenden Artikelgebrauch gefördert wurde, ist ein strittiger Punkt: Einerseits konnten

\footnotetext{
14 Der definite Artikel bzw. das Demonstrativum daz bei NIs bzw. Gerundien ist im 9. Jh. bereits mit 19 Belegen (Tokens) im DDD zu belegen (10. Jh. keine Belege im DDD). Die Zahlen wurden hier nicht in die Graphik integriert, da zu dieser Zeit zwischen entstehendem NI und dem Gerund nicht zu unterscheiden ist (cf. vorheriger Abschnitt). Die absoluten Zahlen (in Tokens) aus DDD und REM der NIs mit definitem Artikel in Relation zu allen DPs mit definitem Artikel des jeweiligen Jahrhunderts sind: 9/2749 im 11. Jh., 21/27712 im 12. Jh., 147/39827 im 13. Jh. sowie 96/30537 in der ersten Hälfte des 14. Jh. Beim indefiniten Artikel betragen die Belegzahlen (Tokens) jeweils: 0/157 im 11. Jh., 8/2677 im 12. Jh., 38/5313 im 13. Jh. sowie 77/4813 in der ersten Hälfte des 14. Jh.
} 
Infinitive bereits syntaktisch nominal (Typ: Irren ist menschlich) gebraucht werden. Als abstrakte Verbalnomen sind diese der Kombination mit definitem Artikel aufgrund der inhärenten Indefinitheit von Abstrakta (cf. Vogel 1996) zunächst avers bzw. beginnt die Grammatikalisierung des definiten Artikels bei Konkreta (cf. z. B. Leiss 2000). Das frühere Auftreten der NIs mit definitem als mit indefinitem Artikel ist also einerseits Ansätzen einer zunehmenden Grammatikalisierung des definiten Artikels, deren Setzung sich auf Abstrakta auszuweiten beginnt, zu verdanken. Andererseits ist aber auch der Anstieg des definiten und indefiniten Artikels ab dem 13. Jh. zu erkennen (cf. Abbildung 3), wobei ab dem 12. Jh. der Gebrauch des NIs mit dem indefiniten Artikel den mit dem definiten überholt. Der Befund ist in Übereinstimmung mit der Literatur (Kloocke 1974, Paul 2007: 313). Da der Gebrauch des indefiniten Artikels mit dem NI im Gegenwartsdeutschen aufgrund der partitiven Nebenlesart des indefiniten Artikels in Kombination mit Massennomina deutlich seltener auftritt (wie bei ?ein Lesen des Buches, cf. Lübbe/Trott 2017: 303), stellt sich neben der Kombination von NI und definitem Artikel also auch die Frage nach der Ursache der „umgekehrten“ Distribution des Mhd.

Der Infinitivforschung ist dabei früh (cf. Jolly 1873: 174) aufgefallen, dass mhd. NIs mit indefinitem Artikel überwiegend in rhematischer Position auftreten. Dort wird typischerweise neue Information kodiert (zum Forschungsüberblick cf. bspw. Coniglio/Schlachter 2015), so z. B. auch im Iwein (3a), im Nibelungenlied (3b) oder bei Johanns von Würzburg Wilhelm von Österreich $(3 \mathrm{c})$.

(3a) hie huop sich ein strîten

(,Nun begann ein Kämpfen/ein Kampf')

(3b) dô gieng ez an ein zücken

(Hartmann von Aue, Iwein: 1021)

der swerte von den sîten.

(,Da kam es zu einem Zücken

der Schwerter von den Seiten')

(Gottfried von Straßburg, Tristen und Isolt: 6866-6867, zitiert nach BMZ)

(3c) daz von den lueten wart ain clagen

(,dass es von den Leuten ein Klagen gab')

(Johann von Würzburg, Wilhelm von Österreich: 10560)

Typischerweise (cf. 3) handelt es sich um existenzielle Sätze mit einem Simplexverb und mit einem NI in rhematischer Position. Die Sätze werden typischerweise von einer Konjunktion, die einen neuen Handlungsbeginn (wie hie in 3a, dô in 3b) anzeigt, eingeleitet. Erst durch den Gebrauch als Subjekt kann der NI thematisch aufgegriffen werden, was typischerweise im Deutschen stark korreliert mit der (typischerweise definiten) Subjektsposition. Unabhängig von der Wahl des jeweiligen Determinators bezeichnet der NI dabei die Verbalhandlung als solche, d. h. Generizität, und drückt nicht mehr den reinen Verlauf der Handlung aus (Pottelberge 2004: 329). Zu der semantischen Änderung nach mhd. Zeit passt, dass erst ab spätmhd. Zeit die syntaktische Koordination des NIs mit einem Substantiv syntaktisch möglich wird (cf. hierzu auch im nächsten Abschnitt Beispiel 6).

Strenggenommen ist im Mhd. die Markierung eines NI mit dem indefiniten Artikel wie in (3) redundant, da der NI als Abstraktum inhärent indefinit ist. Im Mhd. jedoch kann der indefinite 
Artikel neben seiner heutigen Bedeutung auch noch seine ursprüngliche, die der Partitivität, zum Ausdruck bringen (Presslich 1998), was heute bspw. noch im Bairischen nachvollzogen werden kann, cf. ein Kaas ,ein Stück Käse‘ (Kolmer 1999). Da NIs transnumeral, d. h. Massennomina, sind (cf. bspw. Ehrich 1991), erzeugt die Kombination mit dem indefiniten Artikel hier noch eine Partitivitätslesart. Daher kann der NI in (3a) auch mit „ein Stück Kämpfen“, d. h. „ein Kampf", wiedergegeben werden. Zu der Interpretation passt der Befund Demskes (2000: 373f.), wonach neben -ung-Nominalisierungen auch deverbale Nullsuffigierungen (wie nhd. Kampf, Streit, Dreh, mhd. prisbejag) vom NI (prisbejagen, 15. Jh.) ersetzt wurden. Dieser Ablösungsprozess hält bis in fnhd. Zeit an, wie am fnhd. Beispiel Spiegelfecht/Spiegelfechten (FWB) zu erkennen ist. ${ }^{15}$

Unter der Zusammenschau des diachron frequentesten, deverbalen Nominalisierungsmittels für Abstrakta soll daher im folgenden Abschnitt eingehender beleuchtet werden, was dazu führte, dass der wortartunterspezifizierte Infinitiv nominal ist bzw. das häufigste Nominalisierungsmittel für deverbale Abstrakta wurde.

\section{Sprachwandel innerhalb der Nominalisierungsmuster}

Im Gegenwartsdeutschen sind die NIs das häufigste deverbale Nominalisierungsmuster für Abstrakta ohne morphologische Restriktionen (cf. Abschnitt 1), während die -ung-Nominalisierung seit fnhd. Zeit zunehmend Restriktionen auf formaler sowie semantischer Ebene unterliegt (Vogel 1996: 249, Demske 2002). Seit fnhd. Zeit sind -ung-Nominalisierungen immer häufiger mit Basen von perfektiven Präfix- und Partikelverben anzutreffen, während NIs die Aufgabe der Nominalisierung verbaler Simplizia übernehmen. Die Restriktionen der -ung-Nominalisierung setzen sich im Gegenwartsdeutschen fort (zu Details cf. z. B. Iordăchioaia/Werner 2019), cf. (4):

(4a) *Gießung - (das) Gießen, aber Begießung / (das) Begießen

(4b) *Lobung - (das) Loben, aber Verlobung / (das) Verloben

(4c) *Leuchtung - (das) Leuchten, aber Beleuchtung / (das) Beleuchten

Der Prozess der kontinuierlichen Ersetzung der -ung-Nominalisierung durch den NI beginnt also in fnhd. Zeit, wobei beide Nominalisierungsmuster zunächst parallel nebeneinander auftreten (Demske 2002: 87). Nach eigenen Korpusrecherchen lassen sich sogar bereits im späten Mhd. kontextuell parallel auftretende Formen entdecken, cf. (5) aus dem Engelthaler Schwesternbuch aus dem 14. Jh.:

\footnotetext{
${ }^{15}$ Ungleich seltener ist den Recherchen zufolge der umgekehrte Fall eingetreten, bei dem die Nullderivation die Bildung eines NI blockiert. Ein Beispiel hierfür wäre Aderlass neben fnhd. Aderlassen (cf. DTA, FWB). Im Gegensatz zum Englischen überwiegt im Deutschen diachron die Tendenz des Abbaus von Nullsuffigierungen (cf. auch Vogel 1996), da stattdessen Substantive mit punktueller Semantik (genauer: die -er-Nominalisierungen) und im mereologischen Gegensatz zu diesen auch Substantive mit homogener Semantik die NIs, wie in diesem Artikel gezeigt, etabliert werden. Substantive wie ahd. (der) blas ,Puster, Blasvorgang' sind daher heute nur noch in Form ererbter (wie Knall, Dreh, Treff) oder entlehnter Bildungen (wie Chat) belegbar, cf. auch die synchronen Restriktionen (wie (der) *Surf, *Leucht, *Anlehn). Da in den historischen Korpora des Deutschen Nullsuffixe i. d. R. nicht annotiert sind, muss die Ermittlung von Nullsuffigierungen und ihre Bezüge zum NI derzeit ein Forschungsdesiderat bleiben.
} 
(5) Ein swester hiez Gerhus Crumpsitin von Nurenberg, und waz von irn jungen tagen ein siecher mensch, und waz gar andehtig und het vil anvehtung. do unser herre irs leidens ein ende wolt machen, an einem cristabent da sprach sie: [...]

(Engelthaler Schwesternbuch aus dem 14. Jh., zitiert nach MHDBDB 42: 20-23) (,Eine Schwester hieß Gerhus Crumpsit aus Nürnberg, und war in ihrem jungen Alter [bereits] ein kranker Mensch, und war ganz andächtig und hatte viel Bedrängnis. Als unser Herr ihrem Leiden ein Ende machen wollte, an Heiligabend, da sprach sie: [... $]^{\circ}$ )

(Hervorhebung und Übersetzung durch die Autorin)

Da der NI in seiner Funktion als deverbales Nominalisierungsmittel eine deutliche Zunahme im 13./14. Jh. erfährt (cf. Kloocke 1974) und bekannt ist, dass bei der Produktivwerdung neuer Formen sich zunächst alte und neue Formen überlagern (sog. Layering in der Grammatikalisierungstheorie), bevor die neue Form schließlich etabliert ist, ist der frühe Beginn der Produktivwerdung des NIs in der Bereitstellung von Verbalabstrakta vielleicht tatsächlich auf das späte Mhd. zu datieren, was zu der in 3.2 festgestellten Koordinationsfähigkeit von NIs mit Substantiven passt. Hinzu kommt für das Fnhd., dass viele der zu dieser Zeit belegbaren -ung-Nominalisierungen im Gegenwartsdeutschen als NI wiederzugeben sind (wie erschreckung, loßbrennung $\rightarrow$ Erschrecken, Losbrennen, cf. Demske 2002: 14f.). Damit geht eine Zunahme von Restriktionen bei der -ung-Nominalisierung Hand in Hand mit der Zunahme der Produktivität des NIs. $\mathrm{Zu}$ dieser Beobachtung wiederum passt, dass erste Formen der NIs, von lexikalisierten Ausnahmen wie beginnen abgesehen, ausschließlich von imperfektiven Simplizia gebildet werden können, während in der Folge auch NIs zu Präfix- und Partikelverben zu beobachten sind (Behaghel 1923: 356). Es werden also genau die Arten von NIs zuerst produktiv, deren Basen bei der -ung-Nominalisierung zur selben Zeit unproduktiv werden.

Parallel zu den formalen Restriktionen lässt sich auch auf semantischer Ebene die Tendenz feststellen, dass -ung-Nomina immer weniger Aktivitäten oder Prozesse und dafür zunehmend Resultate ausdrücken, d. h. im Sinne einer Lexikalisierung auf eine resultative Semantik reduziert werden, während die NIs die Aufgabe der Kodierung von Prozesssemantik übernehmen, cf. (6):

(6a) Meldung ,Nachricht‘ vs. das Melden ,Prozess/Aktivität, etwas zu melden“

(6b) Einfassung, Mauerwerk' vs. (das) Einfassen von etw. ,Prozess/Aktivität, bei dem/der etwas eingefasst wird“

(6c) Auszeichnung ,Preis` vs. das Auszeichnen ,Prozess/Aktivität, bei dem etwas ausgezeichnet wird`

Wie zu Beginn dieses Abschnitts dargelegt, sind -ung-Nominalisierungen im Gegenwartsdeutschen vor allen Dingen, so überhaupt, zu perfektivischen Basen (Präfixverben wie Zertretung und Partikelverben wie Abtretung) möglich, während Simplizia (*Tretung, *Lachung) mittlerweile mit dem NI nominalisiert werden. Aus theoretischer Sicht liegt es also nahe, dass die ersten NIs auf verbale Simplizia zurückgingen und hier die -ung-Nomina zuerst ablösten.

Um die zugrundeliegenden Basen des NIs morphologisch im Hinblick auf ihre Distribution zu analysieren, wurden alle mhd. NIs in Bezug auf die Morphologie der Basen ausgewertet. Um eine bestmögliche Datenbreite und eindeutige Chronifizierbarkeit zu gewährleisten, fiel dabei 
die Wahl auf das Mhd., da die im REM enthaltenen Texte einerseits eine erste breite Datenbasis für diese Zeitstufe bereitstellen und sich andererseits genügend Texte im REM für diesen Zeitraum datieren lassen, um valide Aussagen treffen zu können. Gemäß Voraussage ist zu erwarten, dass im Mhd. vor allen Dingen Nominalisierungen zu verbalen Simplizia überwiegen, da die -ung-Nominalisierungen in dieser Funktion zuerst Restriktionen unterworfen wurden. Als Präfixverb ist ein Verb dann zu klassifizieren, wenn es morphologisch ein Präfix aufweist (z. B. be- in beladen) und somit in Opposition zu einem verbalen Simplex (z. B. laden) steht. Dieselbe Klassifikationsgrundlage wurde für Partikelverben zugrunde gelegt, nur mit dem Unterschied, dass Partikel im Gegensatz zu Präfixen abtrennbar sein müssen. Um dies zu gewährleisten, wurde jeder Beleg manuell sowohl anhand seiner Belegstelle im REM als auch mithilfe von Lexer überprüft. Um mögliche weitere morphologische Phänomene zu ermitteln, wurde neben der Klassifikation als Simplex, Präfix- und Partikelverb auch geprüft, ob nominalisierte VPs (Typ Kinderkriegen, Teetrinken) oder Lexikalisierungen (Typ Ehebrechen, Befehlhaben) vorkommen. Es wurde nach Tokens (absolute Belegzahlen) und Types (Zusammenfassung der Belege desselben Lexems) ausgewertet, wobei 699 Tokens und 512 Types ermittelt wurden. Zur Ermittlung der NIs wurden folgende grammatische Kriterien für die Klassifikation als NI zugrunde gelegt: Artikelfähigkeit (wie in das Laufen), Auftreten mit Demonstrativ- oder Possessivpronomen (wie in dt. sein/dieses Lachen), Adjektivattribut (wie in dt. schnelles Lesen) oder Genitivattribut (wie in dt. Streichen der Wand im Gegensatz zur VP [[die Wand]-AKK streichen]). Die Ergebnisse der morphologischen Auswertung sind in Abbildung 4 dargestellt.

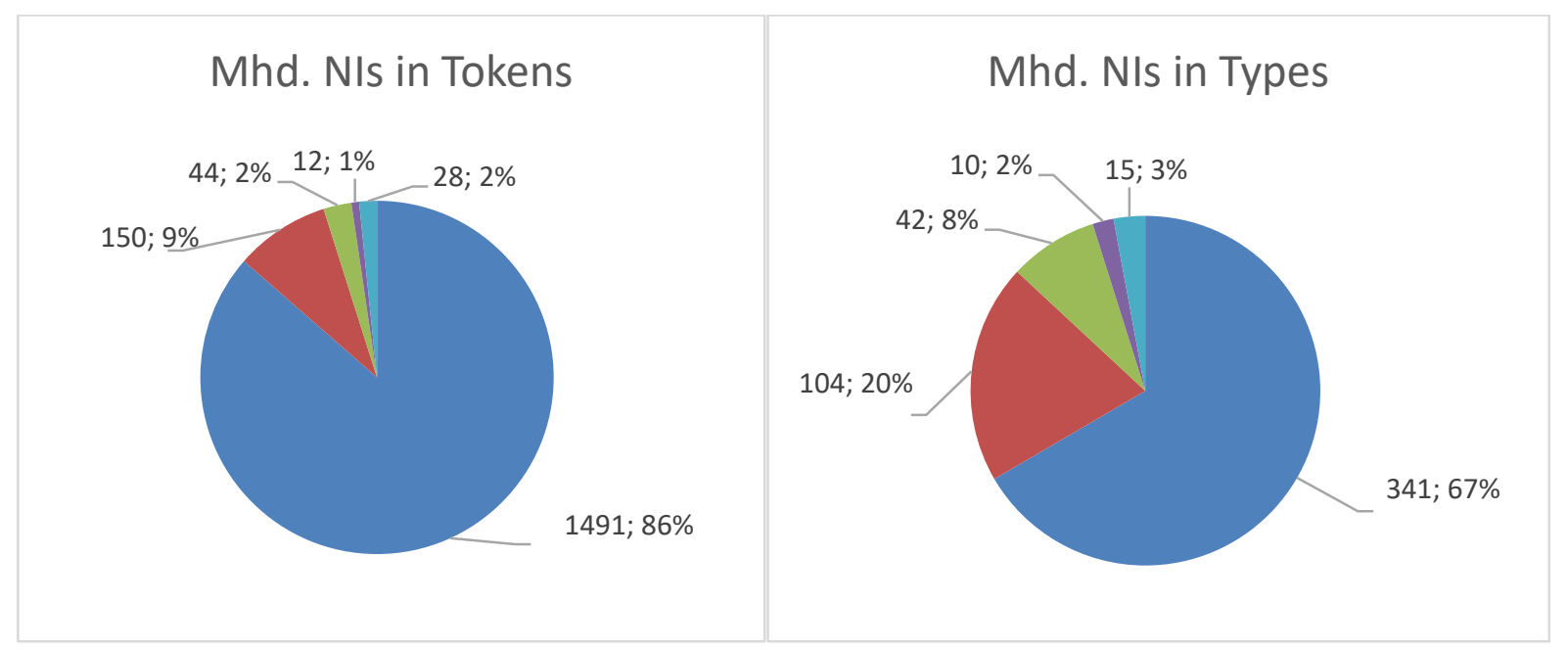

Abbildung 4: Tokens (links) und Types (rechts) des NIs im REM (11.-14. Jh.) ${ }^{16}$

Wie sowohl an Tokens als auch an Types zu erkennen ist, überwiegen im Mhd. die verbalen Simplizia mit 86\% Tokens bzw. 67\% Types (1491 Tokens / 341 Types), gefolgt von den Präfixverben mit 9\% Tokens und 20\% Types (150 Tokens / 104 Types). Gering belegt sind hingegen Partikelverben mit 2\% Tokens bzw. 8\% Types (44 Tokens / 42 Types), marginal hingegen nominalisierte VPs mit 1\% Tokens bzw. 2\% Types (12 Tokens / 10 Types) sowie Lexikalisierungen mit 2\% Tokens bzw. 3\% Types (28 Tokens / 15 Types). Für das Mhd. ist damit festzuhalten, dass sowohl auf Token- als auch auf Type-Ebene bei der Infinitivnominalisierung

\footnotetext{
${ }^{16}$ Die Farben entsprechen den jeweiligen morphologischen Kategorien: blau = Simplex, rot $=$ Präfixverb, hellgrün $=$ Partikelverb, lila $=$ nominalisierte VP und türkis $=$ Lexikalisierung. Die absoluten Zahlen (Types) sind jeweils angegeben.
} 
verbale Simplizia deutlich überwiegen. Präfix- und Partikelverben lassen sich seltener, allerdings trotzdem eindeutig belegen, während andere Formen geradezu marginal sind.

Auch die Diachronie innerhalb des Mhd. ist eine genauere Überprüfung in dieser Hinsicht wert. Bezogen auf die diachrone Entwicklung des Ahd. und Mhd. lassen sich dabei geringere Verschiebungen innerhalb der Basen erkennen. Wie aus Abbildung $5 \mathrm{zu}$ entnehmen, überwiegen in allen Jahrhunderten die Simplizia (hier: Auswertung in Types). Erst ab dem 13. Jh. nimmt der Anteil der Präfixverben, ab dem 14. Jh. zusätzlich der Anteil der Partikelverben kontinuierlich zu.

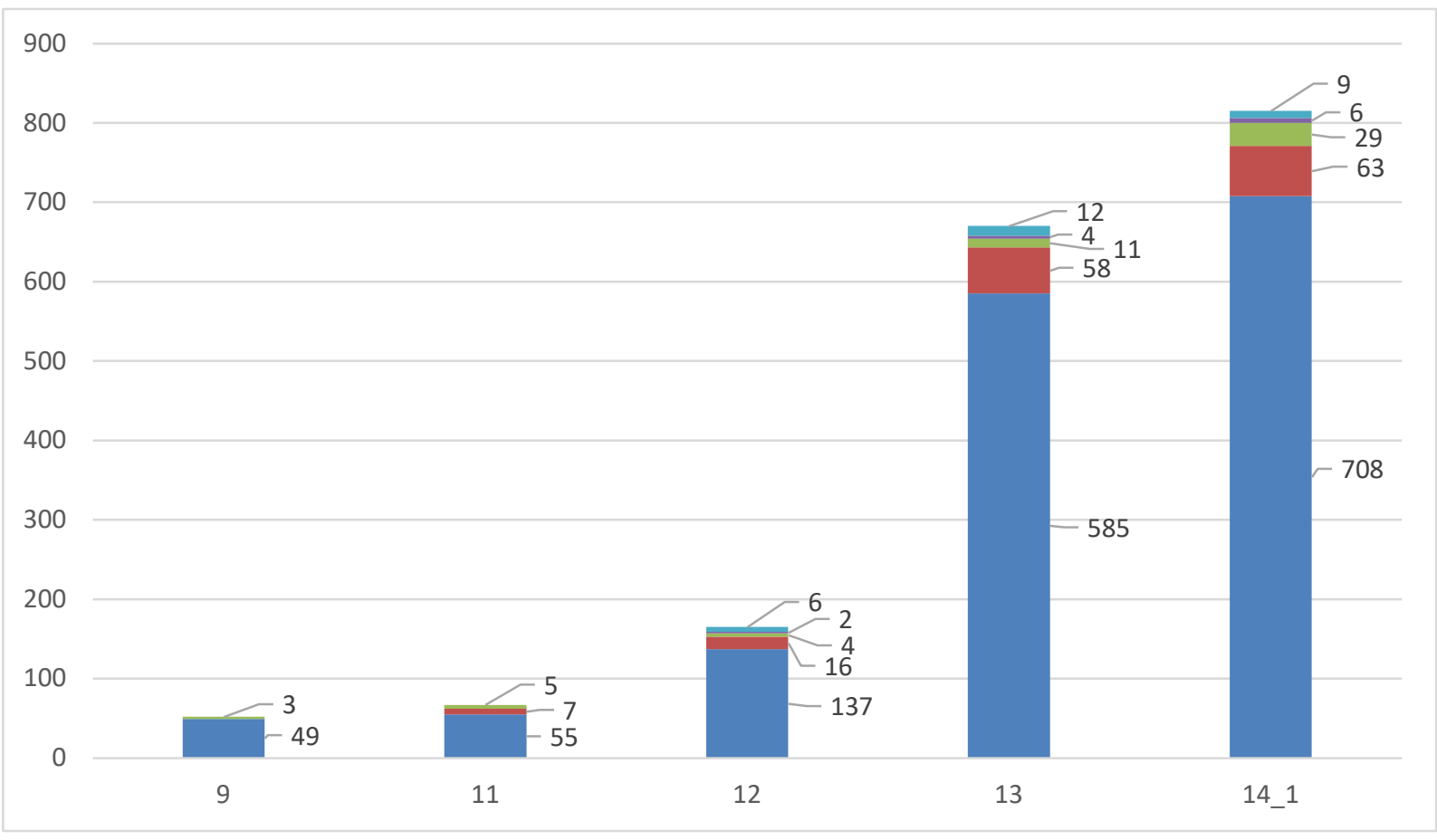

Abbildung 5: Morphologische Analyse der Verben von NIs vom 9. bis 14. Jh. anhand DDD und REM17

Als Zwischenfazit lässt sich festhalten, dass Infinitive verbaler Simplizia zuerst nominalisiert werden, bevor Präfix- und Partikelverben folgen. Dies bestätigt den Befund von Behaghel bzgl. eines Überhangs an Simplizia (Typ: daz dringen), wonach der imperfektive Charakter verbaler Simplizia besonders kompatibel mit der prozessual-kontinuativen Grundsemantik des NIs ist (Behaghel 1906/07: 332). Der Frage, ab wann Infinitivphrasen (und insbesondere VPs) nominalisiert werden konnten, wollen wir uns im nächsten Abschnitt widmen.

\section{$5 \quad$ Nominalisierung von Infinitivphrasen}

Synchron unterliegt die Nominalisierung von Verbalkomplexen aus syntaktischer Perspektive keinen formalen Restriktionen, so dass feststellbare Restriktionen der VP auf syntaktischer Ebene innerhalb der NP fortgesetzt werden, cf. (7; 7a-f aus Haider 2011: 4):

(7a) Alkohol an Jugendliche verabreichen (VP)

(7b) an Jugendliche Alkohol verabreichen (VP)

${ }^{17}$ Legende: blau $=$ Simplex, rot $=$ Präfixverb, hellgrün $=$ Partikelverb, lila $=$ nominalisierte VP und türkis $=$ Lexikalisierung. Die absoluten Zahlen (Types) sind jeweils angegeben. Für das 10. Jh. gibt es abermals keine Belege, da die im REM enthaltenen Texte hier nicht eindeutig chronologisch zuzuordnen (10./11. Jh.) sind. 
(7c) (das) [Verabreichen von Alkohol an Jugendliche] (NP)

(7d) (das) *[Verabreichen an Jugendliche $\mathrm{i}_{\mathrm{i}}$ von Alkohol $\left.\mathrm{e}_{\mathrm{i}}\right]$ (NP)

(7f) (das) *[Verabreichen an Jugendliche $e_{\mathrm{i}}$ des Alkohols $\left.\mathrm{e}_{\mathrm{i}}\right](\mathrm{NP})$

(7g) (das) Alkohol-Verabreichen (an Jugendliche)

(7h) (das) an-Jugendliche-Alkohol-Verabreichen

Sprachhistorisch ist aus morphosyntaktischer Sicht von Bedeutung, welche Formen der VPNominalisierung sich belegen lassen. Es soll im Folgenden (5.1) also zuerst thematisiert werden, ab wann Formen wie $(7 \mathrm{~g}-\mathrm{h})$ gebildet werden konnten. Aus Sicht der Infinitivphrasen ist zudem auch die Nominalisierung besonders von reflexiven Verben von Bedeutung, was in Abschnitt 5.2 Diskussionsgegenstand sein wird.

\subsection{NIs mit Argumentstruktur}

Bei komponierten Basen sind insbesondere die internen Argumente (wie in 7g) synchron prädestiniert, als „Erstelement“ innerhalb der nominalisierten VP zu fungieren (Blume 2004, Bücking 2010). ${ }^{18}$ Daher stellt sich die Frage, ob dies diachron ebenso war und, falls ja, ab wann sich erste Belege ermitteln lassen. Für den Prozess der Phrasennominalisierung hat sich dabei in der synchronen und diachronen Forschungsliteratur der Terminus „Komposition“ eingebürgert. Mit der Wahl dieses Terminus bleibt morphologisch jedoch offen, ob es sich um eine synthetische Komposition (vom Typ Verträge-/Vertragaushandeln zur VP Verträge/einen Vertrag aushandeln), d. h. eine deverbalen Komposition mit zugrundeliegender Argumentstruktur (zur Diskussion cf. hierzu jüngst Iordăchioaia/Alexiadou/Pairamidis 2017), oder eine Determinativkomposition mit einem NI als Kopf (vom Typ Vertragsaushandeln ,Aushandeln eines Vertrags', hier mit Fuge $-s-$ ) handelt.

Da wir bereits festgestellt haben, dass NIs in der Diachronie zuerst ohne komponierte Basenstruktur, also als Simplizia nominalisiert werden, wäre zu erwarten, dass es sich bei den synchronen Formen vom Typ Kaffeetrinken, Blumengießen, Kindererziehen um Syntagmen, die auf VPs zurückgehen, handelt, solange nicht formale Charakteristika, wie z. B. verbale und damit für die Determinativkomposition charakteristische Erstelemente (Typ Drehtür, Inhalierspray), dagegensprächen. Um also terminologische und somit auch morphologische Ambiguitäten zu vermeiden, soll bei Belegen mit inkorporierter Argumentstruktur - in Anlehnung an Gaeta $(1998,1999)$ und Schlotthauer/Zifonun/Cosma (2014) - von synthetischer Komposition (Typ Taubenvergiften, cf. auch 3.1) gesprochen werden.

Die Frage nach einer Diachronie der Basenabfolge ist dabei schon häufiger ins Blickfeld geraten. Allgemein geht man davon aus, dass nach der Nominalisierung von Simplizia, Präfix- und Partikelverben im Mhd. und Fnhd. die Nominalisierung von VPs beginnt (Pavlov 2002: 242). Behaghel (1923: 357) zufolge entwickelt sich diese Fähigkeit im späten Mhd., obgleich oftmals nicht zu entscheiden ist, ob in den vorliegenden Fällen interne Argumente sich auf den Infinitiv beziehen oder schon Teil der nominalisierten VP sind. Ein seltenes Beispiel einer möglichen VP-Nominalisierung des 12. Jh. wäre etwa:

\footnotetext{
18 Zur Frage der synchronen Distribution von Argumenten auf phrasaler Ebene cf. z. B. Blume (2004), Bücking
} (2010) sowie Schlotthauer/Zifonun/Cosma (2014). 
Biben zan claffen ,Bibbern, Zähneklappern“

(Herbort von Fritzlar, Liet von Troye B: M541, 904-914, zitiert nach REM)

Inwieweit in (8) ein NI mit Argumentstruktur zugrunde gelegt werden kann, ist hier umstritten. ${ }^{19}$ Generell wurden jedoch noch im Mhd. interne Argumente als Genitivattribute, d. h. noch auf Phrasenebene, realisiert (Typ: das Essen der Frucht). Selbst bzgl. einer Klassifikation als synthetische Komposition ambige Belege bleiben wie in (8) im Mhd. also marginal, was auch die Datenauswertung des DDD und REM widerspiegelt: Abbildung 5 hat in diesem Zusammenhang bereits gezeigt, dass sich die VP-Nominalisierung mehrheitlich noch nicht etabliert hatte.

$\mathrm{Ab}$ fnhd. Zeit hingegen lassen sich einige Veränderungen in Bezug auf die Nominalisierungsfähigkeit von Phrasen feststellen, die für die früheren Sprachstufen nicht dokumentierbar sind: Während im Mhd. verbale Simplizia und in Folge Präfix- und Partikelverben nominalisiert werden konnten (cf. Kapitel 4) lassen sich ab dem frühen Fnhd. zunehmend auch Phrasen nominalisieren, was eine qualitative Auswertung spätmhd. und fnhd. Datenmaterials zeigt. Besonders ab dem 17. Jh. treten solche Bildungen durchaus regelhaft auf, cf. einige Beispiele in (9) aus dem DTA: ${ }^{20}$

(9a) das brotbrechen (DTA, 1592)

(9b) das Bludvergiessen Christi (DTA, 1602)

(9c) von dem Bierbrawen (DTA, 1579)

Damit lässt sich diachron bestätigen, dass interne Argumente bei einer VP-Nominalisierung bevorzugt werden (zur Synchronie cf. Blume 2004, Bücking 2010). Schlotthauer/Zifonun/Cosma (2014: 271) bezeichnen synthetische Komposita mit internem Argument (auch: Rektionskomposita) daher als Minimalform aller Phrasenkomposita, auf welche letztere syntaxtheoretisch und - vor dem Hintergrund der vorliegenden Daten - auch entstehungsgeschichtlich zurückzuführen sind.

Offenbar korreliert zudem die gesamte Entwicklung von Phrasenkomposita, die mit der Nominalisierung von VPs mit internen Argumenten ihren Ausgangspunkt nimmt, syntaktisch mit dem Ausbau von OV: So hat das Frz. trotz Möglichkeit der Infinitivnominalisierung bis ins 13 . Jh. nie diese Fähigkeit erworben, sondern die NIs parallel zur Entwicklung von VO sogar abgebaut (cf. Sleeman 2010). Für den Zusammenhang zwischen OV und der Entwicklung von Nominalisierungsmustern mit Argumentstruktur im Deutschen spricht, dass auch andere Nominalisierungsmuster im Fnhd. die Fähigkeit zur Nominalisierung von VPs entwickeln (cf. bspw. Demske 2002 zur -ung-Nominalisierung).

Dass im frühen Nhd. die VP-Nominalisierung bereits etabliert war, ist an dem nächsten Beispiel zu erkennen: In (10 tritt ein NI mit Argument mit koordiniertem Kopf im Rahmen einer Überschrift eines Gesetzestexts auf:

\footnotetext{
${ }^{19}$ Nach eigener Recherche wird in der lexikographischen Tradition im konkreten Fall hier tatsächlich eine phrasale Analyse abgelehnt und eine Analyse als Determinativkomposition angenommen, da auch eine entsprechende -ung-Nominalisierung in Lexer belegt ist (zant-klaffunge). Der Beleg wurde aufgrund dessen in Abbildung 5 daher entsprechend klassifiziert. Als Argument kann man eine Inkorporation eines dadurch transnumeral werdenden Nomens annehmen, vergleichbar mit rad-in radfahren.

${ }^{20}$ Die Suchabfrage im DTA mit definitem Artikel lautete@das *en WITH $\$ p=N N$.
} 
(10) Von heimlichen Kindhaben und tödten durch ihre Mutter. (DTA, 1693)

Die Präposition Von in (10) erscheint hier entgegen dem Gegenwartsdeutschen, wo Vom grammatisch wäre, unklitisiert. Der zweite NI, der mit dem ersten durch und koordiniert ist, teilt sich das interne Argument (hier Kind-) mit dem ersten NI. Dass tatsächlich interne Argumente und keine Erstelemente von Determinativkomposita vorliegen, zeigen insbesondere Belege, bei denen das erhaltene Flexiv innerhalb des NIs (hier -e-) vom Fugenelement zweier im selben Kontext vorhandener Determinativkomposita (hier Krieges- in krieges-leute, krieges-art) abweicht, cf. (11): 21

(11) weil aber man von solchen regeln in dem krieg kaum höret/ ja krieges-leute/ wo man sie dazu obligiren wolte/ ein solches vor die höchste alberkeit achten würden/ so bekenne/ wie ich das kriege-führen an sich selbs dem göttlichen gesetz auf obige maa $\beta$ nicht zuwider achte/ daß doch in der heutigen krieges-art kaum etwas finde/

(DTA, 1700, Hervorhebungen durch die Autorin)

Der häufigste Fall allerdings sind Syntagmen, bei denen ab dem 17. Jh. die Präposition noch getilgt wird, cf. (12):

(12a) das Mühlengehen (DTA, 1604)

(12b) das kirchengehen (DTA, 1628)

(12c) das Gottsschwören (DTA, 1617)

Im 18. Jh. finden sich erstmals NIs mit einem Abstraktum als Erstelement. Deren zugrundeliegende VP wird zuerst kontextuell eingeführt (13a), bevor schließlich die nominalisierte VP wenige Zeilen weiter unten anaphorisch wiederaufgenommen wird, cf. (13b):

(13a) fo werdet ihr ruhe finden vor eure feele [...] (DTA, 1700)

(13b) [...] das ruhe-finden für die seele eine geistliche und ewige verheissung ist [...] (ibid.)

Die Nominalisierung wie in (13b) ist hier ein Fall von anaphorisch eingeführter, komprimierter Syntax. Besonders ab dem 19. Jh. scheint die morphosyntaktische Bandbreite solcher Formen zuzunehmen, was an der Distribution von pronominalen (14a), idiomatisierten (14b) oder adverbphrasalen (14c) Erstelementen abzulesen ist:

(14a) kein blosses Darandenken (DTA, 1845)

(14b) das Zustandekommen (DTA, 1824)

(14c) das Zuhochhalten (DWDS-Kernkorpus, 1955)

\footnotetext{
${ }^{21}$ Da bei der Ermittlung von NIs hier nur Einzelfälle behandelt wurden, wäre eine Untersuchung für alle Nominalisierungstypen (Transposition und Suffigierung) wichtig, um diese Fälle der synthetischen Komposition von der Determinativkomposition für die gesamte Diachronie des Deutschen systematisch unterscheiden zu können. Dies trifft auch für die Ermittlung von Syntagmen mit kausativer Semantik zu wie etwa bei das lebendigmachen (DTA, 1592), die etwa zur selben Zeit wie die internen Argumente belegbar sind, allerdings für NIs alleine nur punktuell auftreten. Hier wäre es für alle Nominalisierungstypen zusammengenommen spannend zu untersuchen, inwieweit dieser Typ tatsächlich systematisch auftritt und welche Generalisierungen sich daraus im Hinblick auf die Dynamik beider Wortbildungstypen, aber auch für die synchrone Unterscheidung von Fugenelementen vs. Flexiven ableiten lassen.
} 
Im Gegenwartsdeutschen ist die Nominalisierung von VPs so weit vorangeschritten, dass bspw. auch definite Artikel des internen Arguments sowie Adverbien als Teil des NIs erscheinen können, cf. (15).

(15a) das laute Nagel-in-die-Wand-Hämmern störte ihn sehr (zitiert nach Lawrenz 2006: 182)

(15b) das laut-Nägel-in-die-Wand-Hämmern störte ihn sehr (ibid.)

(15c) dieses ewige Sich-in Einzelheiten-Verlieren (zitiert nach Lawrenz 2006: 70)

(15d) das Teuren-Wein-in-Flaschen-Abfüllen (zitiert nach Lawrenz 2006: 186)

Weitere Beispiele zu solchen phrasalen Komposita finden sich in Hentschel (2017). Auch wenn mit synthetischen Komposita typischerweise suffigierte Nominalisierungen vom Typ Fliesenleger bezeichnet werden, so ist diese Einengung auf die Notwendigkeit eines Suffixes strenggenommen falsch. Synthetische Komposita benötigen kein Suffix zum Wortartwechsel, sie reichern aber die Bildungen mit bestimmten Merkmalen (wie Genus, Pluralisierbarkeit) bzw. damit einhergehenden semantischen Dimensionen (wie Abstraktheit vs. Singulativität, cf. Werner 2012), also sog. „semantischer Modifikation“, an. Letztere ist ein notwendiges Kriterium der Suffigierung, zusammen mit der Fähigkeit zur Transposition. NIs teilen mit der Suffigierung das Merkmal der Fähigkeit zur Transposition. Daher sind sie als Transpositionen dem ,syntaxbasierten“ Prozess der synthetischen Komposition zugänglich. Vogel (1996: 247) fasst es so:

Durch die Übernahme der syntaktischen Funktion einer fremden Wortart hat ein Element natürlich auch an dieser anderen Klasse Anteil und besetzt dort bestimmte Bereiche. Im System der Wortart besteht also fu $\mathrm{n} \mathrm{kt}$ i o $\mathrm{n}$ a 1 kein prinzipieller Unterschied zwischen Wortbildung und syntaktischer Umkategorisierung.

(Vogel 1996: 247)

In Bezug auf Wilmanns' (1896) Ausführungen zur Wortschatzerweiterung durch den Infinitiv 22 weist sie darauf hin, dass bestimmte funktionale Lücken bzw. „leere Felder“ innerhalb der Wortart durch Transpositionen geschlossen werden. Dies wiederum hängt davon ab, welche semantischen Bereiche das Sprachsystem (d. h. die Sprecher) favorisiert. Unter dieser Perspektive stellt sich auch die Frage, welche Formen des NI das Sprachsystem als nominalisierbar erachtet bzw. was überhaupt als nominalisierbar erachtet werden kann. Daher soll im folgenden Abschnitt die Frage der Reflexivität diskutiert werden.

\subsection{Nominalisierung von reflexiven Verben}

Gemäß Literatur stellten reflexive Verben länger als andere Verben eine Hürde für die Nominalisierung dar, so dass vor mhd. Zeit keine reflexiven Verben nominalisiert wurden (Behaghel 1923: 357), was in einigen im Gegenwartsdeutschen ererbten Belegen noch nachzuvollziehen ist, cf. (16):

(16a) Die Kinder benehmen sich gut.

(16b) *Das Sich-Benehmen der Kinder ist gut.

(16c) Das Benehmen der Kinder ist gut.

22 „Denn wenn auch diese Übergänge nicht durch ableitende Suffixe bezeichnet werden, so treten die Wörter doch dadurch in andere formale Gruppen und nehmen an deren Charakter teil“" (Wilmanns 1896: 24, cf. auch Vogel 1996: 247). 
Auch in den Erhebungen des DDD und REM kommen keine NIs zu reflexiven Verben vor. Während im Gegenwartsdeutschen die Nominalisierung der VP mit Reflexivum ab dem 18. Jh. belegt ist, wurde das Reflexivum bei der Infinitivnominalisierung bis dahin kovert realisiert. Die inhärent verbalen Kategorien Tempus, Genus Verbi und Modalität wurden im Mhd. am Infinitiv zunächst nicht realisiert, d. h. es lagen unterspezifizierte Formen vor (cf. Paul 2007: 312 , hieraus auch 17$):^{23}$

(17a) sît Akers niht will erwinden, sô ist bezzer schern dann schinden (,[...] besser Scheren als Gehäutet-Werden')

(Freidank, Bescheidenheit, 155: 20 zitiert nach Paul 2007: 312)

(17b) wer gesah ie hirz zewürken sô?

(,Wer sah je ein Hirsch-Zerlegt-Werden / (einen) Hirsch Zerlegt-Werden auf diese Art') (Gottfried von Straßburg, Tristan, 2796 zitiert nach Paul 2007: 312)

Als weiteren Grund kann man anführen, dass Reflexivpronomina als ein Objekt ohne konzeptuelle Anbindung fungieren (Haider 2004: 86). Die Fähigkeit zur Nominalisierung eines Infinitivs mit Reflexivpronomen setzt also die Fähigkeit zur Nominalisierung von VPs mit Argumenten voraus. Diese wurde aber erst im Fnhd. entwickelt (cf. vorheriger Abschnitt). Die reflexive Semantik im Mhd. kam also noch inferenziell zustande. Da das Reflexivum heute mitnominalisiert werden kann, gelten reflexivpronomenlose Bildungen (wie Benehmen, Verhalten, cf. Beispiel 16) für das Gegenwartsdeutsche als lexikalisiert (Sandberg 1976: 59). NIs lassen aufgrund ihrer Massennomen-Semantik, die im Gegensatz zu den -ung-Nominalisierungen keine resultative Komponente beinhaltet, im Gegenwartsdeutschen in Analogie zur englischen -ing-Form (cf. hierzu Alexiadou et al. 2013: 85) keine reflexive Interpretation zu, was auch für die Reziprok-Interpretation gilt (Werner 2012: 78; hieraus auch 18):

(18a) Das neue Gerät im Weltall macht eine Ausrichtung der Reflektoren möglich.

,Die Reflektoren werden ausgerichtet' (Patienslesart)

,Die Reflektoren richten sich selbst auf etwas (z. B. Sonnenstand) aus ‘ (Agenslesart) ,Die Reflektoren richten sich zueinander aus' (Reziproklesart)

(18b) Das neue Gerät macht ein Ausrichten der Reflektoren möglich.

,Die Reflektoren richten sich selbst auf etwas (z. B. Sonnenstand) aus‘ (Agenslesart)

,Die Reflektoren werden ausgerichtet' (Patienslesart)

*,Die Reflektoren richten sich zueinander aus' (Reziproklesart)

Die Explizierung von Reflexivität bzw. Reziprozität, d. h. die Nominalisierung von unechten und echten reflexiven Verben, lässt sich erst ab dem frühen Nhd. belegen. Beispiele für unechte reflexive Verben finden sich in (19):

(19a) das Sichselbstbilden (DTA, 1785)

(19b) das Sicherzählenlassen (DTA, 1826)

(19c) das Sich-vorwärts-strecken des ganzen Menschen nach Einem Ziele (DTA, 875)

\footnotetext{
${ }^{23}$ Ein vergleichbarer Fall eines ererbten NI aus dem Gegenwartsdeutschen wäre die Äußerung „Das Entsetzen war groß.“, was nach produktiven Regeln des Gegenwartsdeutschen mit „Das Entsetzt-Sein war groß.“ wiederzugeben wäre.
} 
Echt reflexive Verben finden sich bei (20):

(20a) [...]ja das Sichdummstellen haben zu allen Zeiten, wie jetzt, Viele zu solcher Virtuosität gebracht, [...] (DTA, 1838)

(20b) Man wird das Sichübersichselbsterheben der Seele nicht für einen fehlerhaften Zirkel halten können, weil [...]. (DTA, 1900)

(20c) So ist z. B. das Sichaussprechenkönnen eines der mächtigsten Dämpfungsmittel der Affekte. (DTA, 1900)

Die Nominalisierung reflexiver Verben im 18. Jh. folgt chronologisch der Nominalisierung von Infinitiven mit Argumentstruktur, welche im 16. Jh. gemäß manueller Sichtung des DTA ihren Ausgangspunkt nimmt. Im Folgenden wird ausgeführt, dass die Nominalisierung verbaler Periphrasen chronologisch dazwischen liegt, nämlich im 17. Jh. Damit ist die eingangs aufgeworfene Frage verbunden, was den Ausschlag für die Nominalisierung reflexiver Verben gab: die Fähigkeit zur Nominalisierung von Infinitiven mit Argumentstruktur oder von Verbalperiphrasen. Durch die Nominalisierung von Infinitiven mit Argumentstruktur entstand ein Druck in Bezug auf die reflexiven Verben, die mit ihren Pronomina als Objekten ohne konzeptuelle Anbindung den VPs folgten. Da reflexive Verben häufig als Äquivalente zu Diathesen fungieren können (cf. Das Fahrrad fährt sich gut./Das Fahrrad kann gut gefahren werden.), wurde m. E. über die Integration der Argumentstruktur in die Infinitivnominalisierung der Weg für die Nominalisierung der Verbalperiphrasen (Typ: das Besucht-Haben, das Besucht-Worden-Sein) geebnet, einer Entwicklung, die aufgrund der Fülle der beteiligten Phänomene (Tempus, Genus Verbi, Modalität) jedoch einer eigenen Untersuchung vorbehalten bleiben soll. Festzuhalten bleibt an dieser Stelle: Der NI kann im Gegenwartsdeutschen hinsichtlich Tempus und Modalität (das Gegessen-Haben, Besuchen-Müssen) sowie Genus Verbi (das Gefahren-Werden) spezifiziert sein. Diese verbalen Kategorien gelten als aus der Basis, also dem Infinitiv „Präsens“، abgeleitet (Schlotthauer/Zifonun/Cosma 2014: 257). Historisch war der Infinitiv Präsens diesbzgl. also noch unterspezifiziert (Jolly 1873: 23). Sollte Tempus, Genus Verbi oder Modalität explizit gemacht werden, wurde bevorzugt ein dass-Satz gebildet (Jolly 1873: 175).

Im folgenden Kapitel wollen wir uns nun noch der Semantik der NIs widmen, denn, wie bereits in Kapitel 1 thematisiert, macht bereits Behaghel (1923: 357) darauf aufmerksam, dass NIs nur dann als ,echte“ Nomina gelten, wenn sie wie ein Nomen verwendet werden. Das ist aus lexikalischer Sicht einerseits dann der Fall, sobald der NI im Deutschen nicht mehr auf das dazugehörige Verb rückführbar ist (wie Einkommen ,Gehalt‘ oder Treffen ,Sitzung'). Neben dem lexikalischen Argument, dass bspw. Einkommen nichts mehr mit der ursprünglichen Bedeutung des Verbs (hin)einkommen, eintreten' zu tun hat, kann man andererseits ein grammatisch-semantisches Argument für die Lexikalisierung anführen: Ein Großteil der NIs im Gegenwartsdeutschen ist pluralisierbar, was der prozessualen Semantik der Transpositionen zuwiderläuft (cf. Ehrich 1991, Lübbe/Trott 2017). Mit der Auffassung, dass NIs als „Nomina“ verwendet werden, wird implizit vorausgesetzt, dass Nomina im Deutschen typischerweise zählbar sind, was - bspw. mit Blick auf die transnumeralen Stoffnomina wie Milch, Staub, Puder - nicht der Fall ist. Allerdings lassen sich anhand der Änderung des Pluralisierungsverhaltens wichtige Hinweise auf eine synchrone Lexikalisierung finden, die in früheren Sprachstufen so nicht gegeben ist. 


\section{Zur Lexikalisierung von NIs}

Im Mhd. lassen sich in den Daten, bis auf wenige Ausnahmen ererbter Gerundien (Essen, Trinken, Wissen, Leben), keine semantischen Veränderungen belegen. Die zählbaren NIs des Gegenwartsdeutschen drücken hingegen auch Resultate oder Ereignisinstanzen (wie ein (bestimmtes) Rennen) aus. Als Resultate und Ereignisinstanzen sind NIs häufig auch zählbar (21a-b), auch wenn Zählbarkeit keine notwendige Bedingung für die resultative Semantik ist, was man an der konkretisierten Stoffnomen-Semantik ersehen kann (21c).

(21a) Die Familie hat zwei Einkommen.

(21b) Die drei heutigen Abfahrtsrennen in Kitzbühel.

(21c) Hans legte das Essen auf den Tisch.

Doch wie kommt bei ursprünglich kontinuativen NIs eine entsprechende Lexikalisierung zustande? Wie Iturrioz (1982) am Beispiel der -ung-Nominalisierung als Erster gezeigt hat, konkretisieren Nominalisierungen (sog. coercion) durch den Gebrauch als Argument in einem Satz wie in (22, zitiert nach Iturrioz 1982: 55):

\section{Der anhaltende Regen hat zu Überflutungen geführt.}

Neben den theoretischen Argumenten lässt sich auch empirisch der Anteil lexikalisierter NIs im Gegenwartsdeutschen dokumentieren. Um dies zu erreichen, wurden alle NIs $(\mathrm{n}=2178$ Tokens) des Korpus AMC auf Basis von zwei randomisierten Stichproben ${ }^{24}$ aus dem Jahr 2017 ermittelt und die Belege drei semantischen Kategorien, nämlich Aktivität (auch: Prozess wie das ständige Klopfen), Ereignisinstanz (cf. 38b) und Resultat (cf. 38a) zugeordnet. Die Ergebnisse der Auswertung finden sich in Abbildung 6 dargestellt.

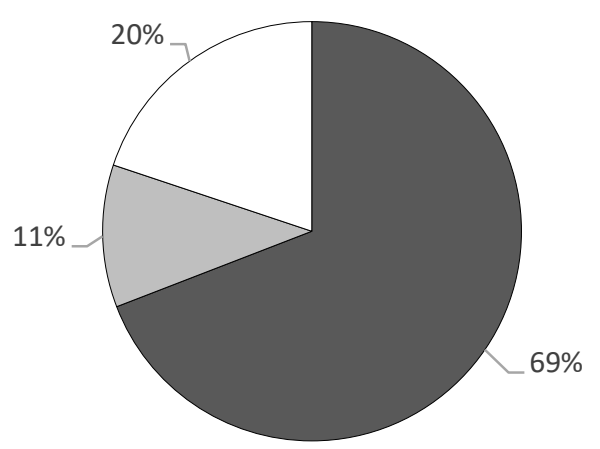

口Aktivität

$\square$ Ereignis

$\square$ Resultat

Abbildung 6: Semantische Auswertung der NIs im AMC nach Token (randomisierte Stichprobe aus dem Jahr 2017)

\footnotetext{
${ }^{24}$ Für die zwei randomisierten Stichproben im AMC (cf. auch Kapitel 1) wurden alle Texte des Jahres 2017 ausgewählt, die mit dem willkürlich gewählten Wort „,der“/,Der“ bzw. „ein“/,Ein“ beginnen (Randomisierung) und mindestens 1 Token enthalten, welches ein Substantiv auf -en mit Genus Neutrum ist. Die Liste der erhaltenen Substantive wurde schließlich manuell kontrolliert und ggf. korrigiert (z. B. *das München), ebenso wurden die bereits vorahd. Lexikalisierungen Essen, Wissen und Leben ausgeschlossen. Auf eine Type-Auswertung wurde in diesem Fall verzichtet, da die ermittelten NIs häufig Polysemien unterworfen sein konnten, was eine kategorienspezifische Zuordnung auf Type-Ebene verunmöglicht hätte.
} 
Wie an Abbildung $6 \mathrm{zu}$ erkennen ist, werden im AMC, was ausschließlich aus Presseerzeugnissen besteht, 69\% (1506 Tokens), also mehr als zwei Drittel, der NIs in ihrer primären Bedeutungsdimension „Aktivität“ (auch: „Prozess“ wie in das Trinken) verwendet, während das knappe restliche Drittel mit 20\% (433 Tokens) der Bedeutungsdimension ,Resultat ${ }^{\text {‘ }}$ (cf. 35c) und 11\% (239 Tokens) der Bedeutungsdimension „Ereignisinstanz“ (cf. 35b) zufällt. ${ }^{25}$ Nimmt die Zahl der prozessualen NIs ab und die der Prozess- und Resultatsbezeichnungen zu, so ist anzunehmen, dass der NI eine ebensolche Lexikalisierung durchläuft wie die -ung-Nominalisierung. NIs sind also, entgegen der Annahme von Iturrioz (1982: 63), prinzipiell genauso anfällig für Lexikalisierung wie andere Nominalisierungsmuster, z. B. die -ung-Derivation (Heizung ,Heizkörper', Bedienung ,Kellnerin'). Wäre es anders, würden die Sprachen der Welt unter denselben Voraussetzungen im Laufe der Zeit immer bei der Infinitivnominalisierung enden und sich nie wieder wandeln.

Die theoretischen und empirischen Befunde passen zu der Annahme, dass Sprache nicht immunisierbar gegen Wandel ist, d. h. ein per se dynamisches System darstellt. Anders formuliert: Nehmen lexikalisierte NIs weiter zu, so dass sie irgendwann überwiegen, so müsste, sofern das System des nominalzentrierten Deutschen aufrecht erhalten bliebe bzw. keine syntaxinternen, nominalisierungenreduzierenden Beschränkungen greifen, ein neues Nominalisierungsmuster vom Sprachsystem generiert werden. Möglich wäre allerdings aus sprachtheoretischer Sicht an dieser Stelle ebenso gut, dass der Nominalstil des Deutschen wie der Scheitelpunkt auf einem morphosyntaktischen Möbiusband zugunsten der Verbalität kippt, d. h. das Deutsche sich hin zu einer verbzentrierten Sprache entwickeln würde (zur Dynamik von Nominalität vs. Verbalität cf. Vogel 1996). Es ist naheliegend anzunehmen, dass den bzgl. der Wortartklassifikation ianusgesichtigen Transpositionen (Infinitive und Partizipien) beim Kippprozess von Nomen auf Verb oder umgekehrt dabei eine Schlüsselrolle zukommt.

\section{$7 \quad$ Zusammenfassung}

Der Artikel behandelte die sprachhistorische Entwicklung des nominalisierten Infinitivs (NI). Dabei wurde empirisch gezeigt, dass die Entwicklung des NIs bei verbalen Simplizia bereits im Ahd. beginnt und das Muster kontinuierlich auf andere Verben sowie ursprünglich verbale Syntagmen ausgeweitet wird.

Als Ursache für die Produktivwerdung des NIs kann nicht der zunehmende Artikelgebrauch, sondern die syntaktische Verwendung von NIs als nominale Konstituenten im Ahd., einhergehend mit einer Abnahme des verbalen $z u$-Infinitivs, sowie der Synkretismus mit den ahd. Gerundien genannt werden. Der Gebrauch mit Determinatoren, v. a. dem definiten Artikel, ist unter dieser Perspektive eher eine Folge, nicht die Ursache der Nominalisierung, auch wenn die Entstehung des definiten Artikels den bzgl. eindeutiger Wortartzuweisung ambigen Infinitiv in Bezug auf seine Wortartenspezifizierung gestärkt haben mag. Definiter Artikel kommt vor indefinitem Artikel in mhd. Zeit, auch wenn letzterer zu dieser Zeit überwiegt. Der NI tritt hier bevorzugt in existenziellen Sätzen, typischerweise in rhematischer Position, auf.

\footnotetext{
${ }^{25}$ Freilich ist, wie in allen korpuslinguistischen Untersuchungen, auch die Abhängigkeit der Textsorte zu berücksichtigen. So dürfte in Presseerzeugnissen der Anteil lexikalisierter NIs (wie Einkommen, Verhalten) höher sein als in anderen, stärker spontansprachlich geprägten Textsorten (wie Chats, Twitter).
} 
Die dokumentierbaren Veränderungen im Hinblick auf eine Komplexitätszunahme der morphologischen Struktur werden im weiteren sprachhistorischen Verlauf ausgebaut. Nachdem zu Beginn im Ahd. imperfektive Simplizia, im Mhd. dann Präfix- und Partikelverben nominalisierbar werden, können ab fnhd. Zeit VPs mit Reflexivpronomina (Typ: das Sich-Waschen) sowie mit internen Argumenten nominalisiert werden. Letztere bilden die Ausgangsbasis für spätere synthetische Phrasenkomposita (Typ: das Äpfelessen). Die diachron wachsende Bedeutung des NI bis zum heutigen Hauptnominalisierungsmittel für deverbale Abstrakta ist auf morphologischer Ebene besonders zu sehen an der Ersetzung bereits existierender, derivativer Nominalisierungsmuster ab spätmhd./fnhd. Zeit, v. a. der -ung-Nominalisierung sowie daneben auch der Nullsuffigierung.

Etwa zur selben Zeit lässt sich als gegenläufige Tendenz jedoch auch die zunehmende Lexikalisierung hochfrequenter NIs mit damit einhergehender Modifikation der inhärenten Prozesssemantik (wie das Laufen, das Herumstehen) feststellen. NIs des Gegenwartsdeutschen können daher, teils kontextspezifisch, auf die Bedeutungsdimensionen Resultat (wie das Essen auf dem Tisch) und Ereignisinstanz (Typ: das Rennen in Kitzbühel) festgelegt sein. Sollten die seit mhd. Zeit bestehenden Lexikalisierungstendenzen so weit zunehmen, dass die Prozesssemantik nicht mehr die primäre ist, so wäre, ebenso wie dies bei den -ung-Nominalisierungen bereits der Fall war, auch hier mit neuerlichen morphosyntaktischen Umkodierungen im Sinne eines Sprachwandels zu rechnen.

\section{Literaturverzeichnis}

Alexiadou, Artemis/Iordăchioaia, Gianina/Schäfer, Florian (2011): "Scaling the Variation in Romance and Germanic Nominalizations." In: Sleeman, Petra/Perridon, Harry (eds.): The Noun Phrase in Romance and Germanic. Structure, variation and change. Amsterdam/Philadelphia: Benjamins: 25-40.

Alexiadou, Artemis (2013): "Nominal vs. verbal ing constructions and the development of the English progressive." English Linguistics Research 2: 126-140.

Alexiadou, Artemis et al. (2013): The realization of external arguments in nominalizations. Journal of Comparative Germanic Linguistics 16: 73-95.

Bech, Gunnar (1955/1957): Studien über das deutsche Verbum infinitum. Kopenhagen: Munksgaard.

Behaghel, Otto (1906/07): „Zum substantivierten Infinitiv.“ Zeitschrift für Wortforschung 8: 329-332.

Behaghel, Otto (1923): Deutsche Syntax. Eine geschichtliche Darstellung. Bd. I. Heidelberg: Winter.

Bierwisch, Manfred (2009): "Nominalization - lexical and syntactic aspects." In: Giannakidou, Anastasia/Rathert, Monika (eds.): Quantification, Definiteness, and Nominalization. Oxford: OUP: 281-320.

Blume, Kerstin (2004): Nominalisierte Infinitive. Tübingen: Niemeyer.

Bopp, Franz (1833): Vergleichende Grammatik des Sanskrit, Zend, Griechischen, Lateinischen, Litthauischen, Gothischen und Deutschen. Berlin: Druckerei der Königlichen Akademie der Wissenschaften, Ferdinand Dümmler. 
Bücking, Sebastian (2010): „Zur Interpretation adnominaler Genitive bei nominalisierten Infinitiven im Deutschen." Zeitschrift für Sprachwissenschaft 29: 39-77.

Coniglio, Marco/Schlachter, Eva (2015): „Das Nachfeld im Deutschen zwischen Syntax, Informations- und Diskursstruktur.“ In: Vinckel-Roisin, Hélène (ed.): Das Nachfeld im Deutschen. Theorie und Empirie. Berlin/Boston: de Gruyter: 141-163.

Dahl, Ingerid/Eroms, Hans-Werner (2014): Kurze deutsche Syntax auf historischer Grundlage. 4. Auflage. Berlin/Boston: de Gruyter.

Demske, Ulrike (2000): „Zur Geschichte der -ung-Nominalisierung im Deutschen. Ein Wandel morphologischer Produktivität.“ Beiträge zur Geschichte der deutschen Sprache und Literatur 122: 365-411.

Demske, Ulrike (2001): „Zur Distribution von Infinitivkomplementen im Ahd.“ In: Linguistische Berichte Sonderheft 9/2001: 61-86.

Demske, Ulrike (2002): "Nominalization and Argument Structure in Early New High German." In: Lang, Ewald/Zimmermann, Ilse (eds.): Nominalization. Berlin: ZASPIL 27: 67-90.

Duden (1995): Der Duden. Grammatik der deutschen Gegenwartssprache. 4. Band. 5. Auflage. Mannheim: Dudenverlag.

Ehrich, Veronika (1991): „Nominalisierungen.“ In: von Stechow, Arnim/Wunderlich, Dieter (eds.): HSK Semantik/Semantics. Berlin/New York: de Gruyter: 441-458.

Ehrich, Veronika/Rapp, Irene (2000): „Sortale Bedeutung und Argumentstruktur. ung-Nominalislerungen im Deutschen.“ Zeitschrift für Sprachwissenschaft 19(2): 245-303.

Engelberg, Stefan (2004): "The Structural Ambiguity of PPs Containing Nominalized Infinitives." In: Moskovsky, Christo (ed.): Proceedings of the 2003 Conference of the Australian Linguistics Society. als.asn.au/proceedings/als2003.html [14.04.2020].

Engelberg, Stefan (2008): Wo man ist, wenn man beim Klettern ist. Anmerkungen zur beimKonstruktion im Deutschen. PPT-Präsentation IDS Mannheim. ids-mannheim.de/filead$\mathrm{min} /$ lexik/lehre/engelberg/Ereignissemantik-Vortrag-pdfs/Engelberg-Mannheim-Dez2008-Ereignissemantik.pdf [01.05.2020].

Erdmann, Oskar (1886): Grundzüge der deutschen Syntax nach ihrer geschichtlichen Entwicklung. Stuttgart: Cotta.

Fuhrhop, Nanna/Teuber, Oliver (2000): „Das Partizip 1 im Deutschen.“ ZAS Papers in Linguistics 16: 100-114.

Gaeta, Livio (1998): "The inflection vs. derivation diachotomy: The case of German infinitives." In: Caron, B. (ed.): Proceedings of the XVI International Congress of Linguists (Paris 20-25, July 1997). Paris: Pergamon: 1-19.

Gaeta, Livio (1999): "Gradienti di nominalizzazione dell'infinitivo Tedesco. Sincronia e diacronia”. Archivio Glottologico Italiano LXXXIV/II: 173-206.

Grimm, Jacob (1826): Deutsche Grammatik. Bd. II. Göttingen: Dieterich.

Gunkel, Lutz/Zifonun, Gisela (2008): “Constraints on relational-adjective noun constructions: A comparative view on English, German and French." Zeitschrift für Anglistik und Amerikanistik 56(3): 283-302.

Hahnfeld, Andrea (2015): Produktivität deverbaler Substantive auf-(er)ei. Quantitative Untersuchung in einem diachronischen Zeitungstext-Korpus. Hamburg: disserta.

Haider, Hubert (2004): „Wie viel Syntax braucht die Semantik, und wie viel Semantik enthält die Syntax?“ Tidsskrift for Sprogforskning 2 (2): 71-90. 
Haider, Hubert (2011): Infinitivnominalisierungen als Testgelände. PPT-Präsentation zum Gastvortrag gehalten am 14.07.2011 an der Universität Bochum. Handout: uni-salzburg.at/index.php?id=24873 [15.04.2020].

Hartmann, Stefan (2014): ““'Nominalization” Taken Literally. A Diachronic Corpus Study of German Word-Formation Patterns." Italian Journal of Linguistics 26, 123-156.

Hentschel, Elke (2017): „Wortbildung, Syntax oder Flexion? Hinweise auf die Entstehung einer neuen Verbalkategorie im Deutschen.“ ZWJW 1 2017/2: 63-88.

Iordăchioaia, Gianina/Alexiadou, Artemis/Pairamidis, Andreas (2017): Morphosyntactic sources for nominal synthetic compounds in English and Greek. In: Meibauer, Jörg/Vogel, Petra Maria (eds.): Special issue on synthetic compounds = Journal of Word Formation 2017.1: 47-71.

Iordăchioaia, Gianina (2019): $D$ and $N$ are Different Nominalizers. PPT-Präsentation, DGfS 2019, Vortrag gehalten am 7. März 2019 an der Universität Bremen.

Iordăchioaia, Gianina/Werner, Martina (2019): "Categorial Shift via Aspect and Gender Change in Deverbal Nouns". In: Cuyckens, Hubert/Heyvaert, Liesbet/Hartmann, Stefan (eds.): Special issue on Categorial shifts: from description to theory and back again. = Language Sciences 73: 62-76.

Iturrioz, José Luis (1982): „Abstraktion: Kategorie und Technik.“ In: Seiler, Hansjakob/Lehmann, Christian (eds.): Apprehension. Das sprachliche Erfassen von Gegenständen. Teil 1: Bereich und Ordnung der Phänomene. Tübingen: Narr: 49-65.

Jolly, Julius (1873): Die Geschichte des Infinitivs im Indogermanischen. München: Ackermann.

Kloocke, Hella (1974): Der Gebrauch des substantivierten Infinitivs im Mittelhochdeutschen. Göppingen: Kümmerle.

Kolmer, Agnes (1999): Zur MASS-COUNT-Distinktion im Bairischen. Arbeitspapier 34 (neue Folge). Universität Köln: Institut für Sprachwissenschaft.

Koning, Wilhelm (1933): „Substantivierte infinite Verbformen mit verbaler Rektion.“ Die neueren Sprachen 41: 90-98.

Lawrenz, Birgit (2006): Moderne deutsche Wortbildung. Phrasale Wortbildung im Deutschen: Linguistische Untersuchung und sprachdidaktische Behandlung. Hamburg: Kovač.

Leiss, Elisabeth (2000): Artikel und Aspekt. Die grammatischen Muster von Definitheit. Berlin/New York: de Gruyter.

Leiss, Elisabeth (2002): „Die Wortart ,Verb“.“ In: Cruse, Alan/Hundsnurscher, David Franz Job, Michael/Lutzeier, Peter Rolf (eds.): HSK 21.1 Lexikologie. Berlin/New York: de Gruyter: 605-616.

Lübbe, Anja/Trott, Isabel (2017): „Zur Bedeutungskonstitution Nominalisierter Infinitive.“ Deutsche Sprache 45: 289-316.

Marchis Moreno, Mihaela (2015). „Relational adjectives as interfaces.“ Studia Linguistica 69(3): 403-332.

Mayerthaler, Willi/Fliedl, Günther/Winkler, Christian (1993): Infinitivprominenz in europäischen Sprachen. Band 1: Die Romania (samt Baskisch). Tübingen: Narr.

Meinschäfer, Judith (2016): „Nominalizations.“ In: Fischer, Susann/Gabriel, Christoph (eds.): Manual of Grammatical Interfaces in Romance. Berlin/Boston: de Gruyter: 391-417. 
Moessner, Lilo (1997): “ing-constructions in Middle English.” In: Fisiak, Jacek (ed.): Studies in Middle English. Berlin/New York: de Gruyter: 335-350.

Panagl, Oswald (2006): „Zur verbalen Konstruktion deverbativer Nomina“. In: Crespo, Emilio/de la Villa, Jesús/Revuelta, Antionio (eds.): Word Classes and Related Topics in Ancient Greek. Proceedings on the Conference on "Greek Syntax and Word classes", held in Madrid on 18-21 June 2003. Louvain-la-Neuve: Peeters.

Paul, Hermann (2007): Mittelhochdeutsche Grammatik. Neu bearb. von Thomas Klein, HansJoachim Solms, Klaus-Peter Wegera. 25. Aufl. Niemeyer: Tübingen.

Pavlov, Vladimir (2002): „Deverbale Nominalisierung im Frühneuhochdeutschen im Vergleich mit dem Neuhochdeutschen. “ In: Habermann, Mechthild/Müller, Peter/Munske, Horst Haider (eds.): Historische Wortbildung des Deutschen. Tübingen: Niemeyer: 227-244.

Presslich, Marion (1998): Partitivität und Indefinitheit. Die Entstehung und Entwicklung des indefiniten Artikels in den germanischen und romanischen Sprachen am Beispiel des Deutschen, Niederländischen, Französischen und Italienischen. Frankfurt: Lang.

Pottelberge, Jeroen van (2004): Der am-Progessiv. Struktur und parallele Entwicklung in den kontinentalwestgermanischen Sprachen. Tübingen: Narr.

Sandberg, Bengt (1976): Die neutrale -(e)n-Ableitung der deutschen Gegenwartssprache, zu dem Aspekt der Lexikalisierung bei den Verbalsubstantiven. Göteborg: Acta Univ. Gothoburg.

Schlotthauer, Susan/Zifonun, Gisela/Cosma, Ruxandra (2014): „Verbale und nominale Infinitive - Strukturelle Eigenschaften und Funktion als Subjekt.“ In: Cosma, Ruxandra et al. (eds.): Komplexe Argumentstrukturen. Kontrastive Untersuchungen zum Deutschen, Rumänischen und Englischen. Berlin/Boston: de Gruyter: 253-282.

Schrodt, Richard (2004): Althochdeutsche Grammatik II: Syntax. Tübingen: Niemeyer.

Sleeman, Petra (2010): "The nominalized infinitive in French: structure and change." Linguística: revista de estudos linguísticos da Universidade do Porto 5: 145-173.

Tanaka, Tomoyuki (2007): "The rise of lexical subjects in English infinitives." Journal of Comparative Germanic Linguistics 10: 25-67.

Vogel, Petra Maria (1996): Wortart und Wortartwechsel. Zur Konversion und verwandten Erscheinungen im Deutschen und in anderen Sprachen. Berlin/New York: de Gruyter.

Weber, William Lander (1899): “The English gerund." Proceedings of Modern Language Association 14: 1xxv-1xxvi.

Werner, Martina (2012): Genus, Derivation und Quantifikation. Zur Funktion der Suffigierung und verwandter Phänomene im Deutschen. Berlin/Boston: de Gruyter.

Wilmanns, Wilhelm (1896): Deutsche Grammatik. Gotisch, Alt-, Mittel- und Neuhochdeutsch. Bd. 2: Wortbildung. Strassburg: Trübner.

Wilmanns, Wilhelm (1906): Deutsche Grammatik. Gotisch, Alt-, Mittel- und Neuhochdeutsch. Bd. 3: Flexion. Strassburg: Trübner.

Zifonun, Gisela (2011). „Relationale Adjektive - ein „klassisches“ Muster im europäischen Vergleich." Deutsche Sprache 9(2): 98-112. 


\section{Korpora, Quellen und Online-Wörterbücher}

$\mathrm{AMC}=$ Austrian Media Corpus. Zugang beim Austrian Centre for Digital Humanities and Cultural Heritage (ÖAW). oeaw.ac.at/acdh/tools/amc-austria-media-corpus/ [01.05.2020].

BMZ = Mittelhochdeutsches Wörterbuch . Mit Benutzung des Nachlasses von Georg Friedrich Benecke ausgearbeitet von Wilhelm Müller und Friedrich Zarncke. 3 Bde. Leipzig 18541866. woerterbuchnetz.de/cgi-bin/WBNetz/wbgui_py?sigle=BMZ [01.05.2020]

DDD = Deutsch Diachron Digital. deutschdiachrondigital.de [01.05.2020].

DTA $=$ Deutsches Text Archiv (1437-1927) dwds.de [01.05.2020].

DWB $=$ Deutsches Wörterbuch von Jacob und Wilhelm Grimm. 16 Bde. in 32 Teilbänden. Leipzig 1854-1961. Quellenverzeichnis Leipzig 1971. dwb.uni-trier.de/de/ [01.05.2020].

DWDS-Kernkorpus $=$ DWDS-Kernkorpus (1900-1999) dwds.de [01.05.2020]

FWB = Frühneuhochdeutsches Wörterbuch. Hgg. von der Akademie der Wissenschaften, Göttingen. fwb-online.de [01.05.2020].

Hartmann von Aue: Iwein. Hg. von Georg Friedrich Benecke und Karl Lachmann. Neu bearb. von Ludwig Wolff. 7. Ausg., Bd. 1: Text, Berlin 1968. mhdwb-online.de/ [01.05.2020].

Johann von Würzburg: Wilhelm von Österreich. Hg. von Ernst Regel (2003). (Deutsche Texte des Mittelalters 3.3). Auflage. Hildesheim: Weidmann.

Lexer = Matthias Lexer: Mittelhochdeutsches Handwörterbuch. 3 Bde. Leipzig 1872-1878. woerterbuchnetz.de/cgi-bin/WBNetz/wbgui_py?sigle=Lexer [01.05.2020].

MHDBDB = Mittelhochdeutsche Begriffsdatenbank. mhdbdb.sbg.ac.at/ [01.05.2020].

Handschriftencensus $=$ handschriftencensus.de [01.05.2020].

$\mathrm{REM}=$ Referenzkorpus Mittelhochdeutsch. Hgg. von der Universität Bochum, Institut für Linguistik. linguistics.rub.de/rem/ [01.05.2020]. 